ANALYSIS \& PDE

Volume $4 \quad$ No. $2 \quad 2011$

TAOUFIK HMIDI

ON A MAXIMUM PRINCIPLE AND ITS APPLICATION TO THE LOGARITHMICALLY CRITICAL BOUSSINESQ SYSTEM

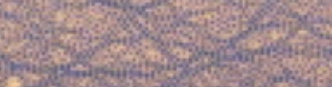




\title{
ON A MAXIMUM PRINCIPLE AND ITS APPLICATION TO THE LOGARITHMICALLY CRITICAL BOUSSINESQ SYSTEM
}

\author{
TAOUFIK HMIDI
}

\begin{abstract}
In this paper we study a transport-diffusion model with some logarithmic dissipations. We look for two kinds of estimates. The first is a maximum principle whose proof is based on Askey theorem concerning characteristic functions and some tools from the theory of $C_{0}$-semigroups. The second is a smoothing effect based on some results from harmonic analysis and submarkovian operators. As an application we prove the global well-posedness for the two-dimensional Euler-Boussinesq system where the dissipation occurs only on the temperature equation and has the form $|\mathrm{D}| / \log ^{\alpha}\left(e^{4}+\mathrm{D}\right)$, with $\alpha \in\left[0, \frac{1}{2}\right]$. This result improves on an earlier critical dissipation condition $(\alpha=0)$ needed for global well-posedness.
\end{abstract}

1. Introduction

2. Notation and preliminaries 251

3. Maximum principle 253

4. Proof of the generalized Bernstein inequality (Theorem 1.3) 264

$\begin{array}{ll}\text { 5. Commutator estimates } & 269\end{array}$

6. Smoothing effects 272

7. Proof of Theorem $1.5 \quad 273$

$\begin{array}{ll}\text { References } & 283\end{array}$

\section{Introduction}

The first goal of this paper is to study some mathematical problems related to the following transportdiffusion model with logarithmic dissipations:

$$
\left\{\begin{array}{l}
\partial_{t} \theta+v \cdot \nabla \theta+\kappa \frac{|\mathrm{D}|^{\beta}}{\operatorname{div}^{\alpha}(\lambda+|\mathrm{D}|)} \theta=0, \quad(t, x) \in \mathbb{R}_{+} \times \mathbb{R}^{d}, \\
\theta_{\mid t=0}=\theta_{0} .
\end{array}\right.
$$

Here, the unknown is the scalar function $\theta$, the velocity $v$ is a time-dependent vector field with zero divergence and $\theta_{0}$ is the initial datum. The parameters are $\kappa \geq 0, \lambda>1$ and $\alpha, \beta \in \mathbb{R}$. The operator $|\mathrm{D}|^{\beta} / \log ^{\alpha}(\lambda+|\mathrm{D}|)$ is defined through its Fourier transform:

$$
\mathscr{F}\left(\frac{|\mathrm{D}|^{\beta}}{\log ^{\alpha}(\lambda+|\mathrm{D}|)} f\right)(\xi)=\frac{|\xi|^{\beta}}{\log ^{\alpha}(\lambda+|\xi|)}(\mathscr{F} f)(\xi) .
$$

We will discuss along this paper some quantitative properties for this model; especially two kinds of information will be established: maximum principle and some smoothing effects. We notice that the

MSC2000: primary 35Q35; secondary 76D03.

Keywords: Boussinesq system, logarithmic dissipation, global existence. 
special case of (1) corresponding to $\alpha=0$ and $\beta \in[0,2]$ appears naturally in some fluid models like quasigeostrophic equations or Boussinesq systems. In this context A. Córdoba and D. Córdoba [2004] established a priori $L^{p}$ estimates: for $p \in[1, \infty]$ and $t \geq 0$,

$$
\|\theta(t)\|_{L^{p}} \leq\left\|\theta_{0}\right\|_{L^{p}} .
$$

We remark also that the proof in the case $p=+\infty$ can be obtained from the following representation of the fractional Laplacian $|\mathrm{D}|^{\beta}$ :

$$
|\mathrm{D}|^{\beta} f(x)=c_{d} \int_{\mathbb{R}^{d}} \frac{f(x)-f(y)}{|x-y|^{d+\beta}} d y .
$$

Indeed, one can check that if a continuous function reaches its maximum at a point $x_{0}$, then $|\mathrm{D}|^{\beta} f\left(x_{0}\right) \geq 0$ and hence we conclude as for the heat equation. Our first main result is a generalization of the result of [Córdoba and Córdoba 2004] to (1).

Theorem 1.1. Let $\kappa \geq 0, d \in\{2,3\}, \beta \in] 0,1], \alpha \geq 0, \lambda \geq e^{(3+2 \alpha) / \beta}$ and $p \in[1, \infty]$. Then any smooth solution of (1) satisfies

$$
\|\theta(t)\|_{L^{p}} \leq\left\|\theta_{0}\right\|_{L^{p}} .
$$

Remark 1.2. The restriction on the parameter $\beta$ is technical and we believe that the above theorem remains true for $\beta \in] 1,2[$ and $\alpha>0$.

We discuss the proof in the special case $v \equiv 0$. Equation (1) is reduced to the fractional heat equation

$$
\partial_{t} \theta+\kappa \mathscr{L} \theta=0 \quad \text { with } \quad \mathscr{L}:=\frac{|\mathrm{D}|^{\beta}}{\log ^{\alpha}(\lambda+|\mathrm{D}|)} .
$$

The solution is explicitly given by the convolution formula

$$
\theta(t, x)=K_{t} \star \theta_{0}(x) \quad \text { with } \quad \widehat{K}_{t}(\xi)=e^{-t|\xi|^{\beta} / \log ^{\alpha}(\lambda+|\xi|)} .
$$

We will show that the family $\left(K_{t}\right)_{t \geq 0}$ is a convolution semigroup of probabilities, which means that $\mathscr{L}$ is the generator of a Lévy semigroup. Consequently, this family is a $C_{0}$-semigroup of contractions on $L^{p}$ for every $p \in\left[1, \infty\left[\right.\right.$. The important step in the proof is to get the positivity of the kernel $K_{t}$. For this purpose we use Askey's criterion for characteristic functions; see Theorem 3.4. The restrictions on the dimension $d$ and the values of $\beta$ are due to the use of this criterion. Now to deal with the full transport-diffusion equation (1) we use some results from the theory of $C_{0}$-semigroups of contractions.

The second estimate that we intend to establish is a generalized Bernstein inequality. Before stating the result we recall that for $q \in \mathbb{N}$ the operator $\Delta_{q}$ is the frequency localization around an annulus of size $2^{q}$; see next section for more details.

Theorem 1.3. Let $d \in\{1,2,3\}, \beta \in] 0,1], \alpha \geq 0, \lambda \geq e^{(3+2 \alpha) / \beta}$ and $\left.p \in\right] 1, \infty\left[\right.$. For $q \in \mathbb{N}$ and $f \in \mathscr{Y}\left(\mathbb{R}^{d}\right)$,

$$
2^{q \beta}(q+1)^{-\alpha}\left\|\Delta_{q} f\right\|_{L^{p}}^{p} \leq C \int_{\mathbb{R}^{d}}\left(\frac{|\mathrm{D}|^{\beta}}{\log ^{\alpha}(\lambda+|\mathrm{D}|)} \Delta_{q} f\right)\left|\Delta_{q} f\right|^{p-2} \Delta_{q} f d x,
$$

where $C$ is a constant depending on $p, \alpha, \beta$ and $\lambda$.

The proof relies on some tools from the theory of Lévy operators or more generally submarkovians operators combined with some results from harmonic analysis. 
Remarks 1.4. (1) When $\alpha=0$ then the inequality above is valid for all $\beta \in[0,2]$. The case $\beta=2$ was discussed in [Danchin 2001; Planchon 2000]. The remaining case $\beta \in$ [0,2[ was treated in [2007], but only for $p \geq 2$.

(2) The proof for the case $p=2$ is an easy consequence of the Plancherel identity and does not require any assumption on the parameters $\alpha, \beta$ and $\lambda$.

The second part of this paper is concerned with an application of Theorems 1.1 and 1.3 to the following Boussinesq model with general dissipation

$$
\left\{\begin{array}{l}
\partial_{t} v+v \cdot \nabla v+\nabla \pi=\theta e_{2}, \quad(t, x) \in \mathbb{R}_{+} \times \mathbb{R}^{2}, \\
\partial_{t} \theta+v \cdot \nabla \theta+\kappa \mathscr{L} \theta=0 \\
\operatorname{div} v=0 \\
v_{\mid t=0}=v_{0}, \theta_{\mid t=0}=\theta_{0} .
\end{array}\right.
$$

Here, the velocity field $v$ is given by $v=\left(v^{1}, v^{2}\right)$, while the pressure $\pi$ and the temperature $\theta$ are scalar functions. The force term $\theta e_{2}$ in the velocity equation, with $e_{2}$ the vector $(0,1)$, models the effect of gravity on the fluid motion. The operator $\mathscr{L}$, whose form may vary, is used to take into account anomalous diffusion in the fluid motion.

From a mathematical point of view, the question of global well-posedness for the inviscid model, corresponding to $\kappa=0$, is extremely hard to deal with. We point out that the classical theory of symmetric hyperbolic quasilinear systems can be applied to this system and thus we can get local well-posedness for smooth initial data. The significant quantity that one needs to bound in order to get global existence is the $L^{\infty}$-norm of the vorticity, defined by $\omega=\operatorname{curl} v=\partial_{1} v^{2}-\partial_{2} v^{1}$. Now we observe from the first equation of (3) that $\omega$ solves the equation

$$
\partial_{t} \omega+v \cdot \nabla \omega=\partial_{1} \theta .
$$

The main difficulty encountered for global existence is due to the lack of strong dissipation in the temperature equation: we don't see how to estimate in a suitable way the quantity $\int_{0}^{T}\left\|\partial_{1} \theta\right\|_{L^{\infty}}$. However, the situation in the viscous case, $\kappa>0$ and $\mathscr{L}=-\Delta$, is well understood, and the question of global existence was solved recently in a series of papers. Chae [2006] proved global existence and uniqueness for initial data $\left(v_{0}, \theta_{0}\right) \in H^{s} \times H^{s}$, with $s>2$; see also [Hou and Li 2005]. This result was improved in [Hmidi and Keraani 2009] to initial data $v_{0} \in B_{p, 1}^{(2 / p)+1}$ and $\theta_{0} \in B_{p, 1}^{-1+(2 / p)} \cap L^{r}, r>2$. The global existence of Yudovich solutions for this system was treated in [Danchin and Paicu 2009]. The same authors [2008] constructed global strong solutions for a dissipative term of the form $\partial_{11} \theta$ instead of $\Delta \theta$. In [Hmidi and Zerguine 2010; Hmidi et al. 2011] we try to understand the lower dissipation $\mathscr{L}=|\mathrm{D}|{ }^{\alpha}$ needed for global existence. In the first of these papers we proved global well-posedness when $\alpha \in] 1,2[$. The proof relies on the fact that the dissipation is sufficiently strong to counterbalance the possible amplification of the vorticity due to $\partial_{1} \theta$. However the case $\alpha=1$ is not reached by this method, and this value of $\alpha$ is called critical, in the sense that the dissipation and the amplification of the vorticity due to $\partial_{1} \theta$ have the same order.

In [Hmidi et al. 2011] we proved there is some hidden structure leading to global existence in the critical case. More precisely, we introduced the mixed quantity

$$
\Gamma=\omega+\mathscr{R} \theta, \quad \text { with } \quad \mathscr{R}:=\frac{\partial_{1}}{|\mathrm{D}|} ;
$$


it satisfies the equation

$$
\partial_{t} \Gamma+v \cdot \nabla \Gamma=-[\mathscr{R}, v \cdot \nabla] \theta .
$$

As a matter of fact, the problem in the framework of Lebesgue spaces is reduced to estimating the commutator between the advection $v \cdot \nabla$ and Riesz transform $\mathscr{R}$, which is homogenous of degree zero. Since Riesz transform is a Calderón-Zygmund operator, we can, using the smoothing effects for $\theta$ in a suitable way, get a global estimate of $\|\omega(t)\|_{L^{p}}$. We can then use this information to control more strong norms of the vorticity, like $\|\omega(t)\|_{L^{\infty}}$ or $\|\omega(t)\|_{B_{\infty, 1}^{0}}$. For more discussion about the global wellposedness problem concerning other classes of Boussinesq systems we refer to [Hmidi et al. 2010; Miao and Xue 2009].

Our goal here is to relax the critical dissipation needed for global well-posedness by some logarithmic factor. More precisely, we will study the logarithmically critical Boussinesq model

$$
\left\{\begin{array}{l}
\partial_{t} v+v \cdot \nabla v+\nabla \pi=\theta e_{2}, \\
\partial_{t} \theta+v \cdot \nabla \theta+\frac{|\mathrm{D}|}{\log ^{\alpha}(\lambda+|\mathrm{D}|)} \theta=0 \\
\operatorname{div} v=0, \\
v_{\mid t=0}=v^{0}, \quad \theta_{\mid t=0}=\theta^{0}
\end{array}\right.
$$

Before stating our result we need some new definitions. We define the logarithmic Riesz transform $\mathscr{R}_{\alpha}$ by $\mathscr{R}_{\alpha}=\left(\partial_{1} /|\mathrm{D}|\right) \log ^{\alpha}(\lambda+|\mathrm{D}|)$. Next, for given $\alpha \in \mathbb{R}$ we define the function spaces $\left\{\mathscr{L}_{p}\right\}_{1 \leq p \leq \infty}$ by

$$
u \in \mathscr{X}_{p} \Longleftrightarrow\|u\|_{\mathscr{X}_{p}}:=\|u\|_{B_{\infty, 1}^{0} \cap L^{p}}+\left\|\mathscr{R}_{\alpha} u\right\|_{B_{\infty, 1}^{0} \cap L^{p}}<\infty .
$$

Our result reads as follows (see Section 2 for the definitions and the basic properties of Besov spaces).

Theorem 1.5. Let $\alpha \in\left[0, \frac{1}{2}\right], \lambda \geq e^{4}$ and $\left.p \in\right] 2, \infty\left[\right.$. Let $v_{0} \in B_{\infty, 1}^{1} \cap \dot{W}^{1, p}$ be a divergence-free vector field of $\mathbb{R}^{2}$ and $\theta_{0} \in \mathscr{X}_{p}$. Then there exists a unique global solution $(v, \theta)$ to the system $(5)$ with

$$
v \in L_{\mathrm{loc}}^{\infty}\left(\mathbb{R}_{+} ; B_{\infty, 1}^{1} \cap \dot{W}^{1, p}\right), \quad \theta \in L_{\mathrm{loc}}^{\infty}\left(\mathbb{R}_{+} ; \mathscr{X}_{p}\right) \cap \widetilde{L}_{\mathrm{loc}}^{1}\left(\mathbb{R}_{+} ; B_{p, \infty}^{1,-\alpha}\right) .
$$

The proof shares the same ideas as the case $\alpha=0$ treated in [Hmidi et al. 2011] but with more technical difficulties. We define

$$
\mathscr{R}_{\alpha}=\frac{\partial_{1}}{|\mathrm{D}|} \log ^{\alpha}(\lambda+|\mathrm{D}|) \quad \text { and } \quad \Gamma=\omega+\mathscr{R}_{\alpha} \theta
$$

Then we get

$$
\partial_{t} \Gamma+v \cdot \nabla \Gamma=-\left[\mathscr{R}_{\alpha}, v \cdot \nabla\right] \theta .
$$

To estimate the commutator in the framework of Lebesgue spaces we use the paradifferential calculus combined with Theorems 1.1 and 1.3.

Remarks 1.6. (1) For global well-posedness for the generalized Navier-Stokes system in dimension three, Tao [2009] proved that we can improve the dissipation $|\mathrm{D}|^{5 / 2}$ to

$$
\frac{|\mathrm{D}|^{5 / 2}}{\log ^{1 / 2}(2+|\mathrm{D}|)} \text {. }
$$


(2) The space $\mathscr{X}_{p}$ is less regular than the space $B_{\infty, 1}^{\varepsilon} \cap B_{p, 1}^{\varepsilon}$, for all $\varepsilon>0$. More precisely, we will see in Corollary 4.3 that $B_{\infty, 1}^{\varepsilon} \cap B_{p, 1}^{\varepsilon} \hookrightarrow \mathscr{X}_{p}$.

(3) If we take $\theta=0$ then the system (5) is reduced to the two-dimensional Euler system. It is wellknown that this system is globally well-posed in $H^{s}$ for $s>2$. The main tool for global existence is the BKM criterion [Beale et al. 1984] ensuring that the development of finite-time singularities for Kato's solutions is related to the blowup of the $L^{\infty}$ norm of the vorticity near maximal time existence. Vishik [1998] extended the global existence of strong solutions to initial data belonging to Besov spaces $B_{p, 1}^{1+2 / p}$. These spaces have the same scale as Lipschitz functions and in this sense they are called critical and it is not at all clear whether BKM criterion can be used in this context.

(4) Since $B_{r, 1}^{1+2 / r} \hookrightarrow B_{\infty, 1}^{1} \cap \dot{W}^{1, p}$ for all $r \in[1,+\infty[$ and $p>\max \{r, 2\}$, the space of initial velocity in our theorem contains all the critical spaces $B_{p, 1}^{1+2 / p}$ except the biggest one, $B_{\infty, 1}^{1}$. For the limiting case we have been able to prove the global existence only under the extra assumption that $\nabla v_{0} \in L^{p}$ for some $p \in] 2, \infty\left[\right.$. The reason behind this extra assumption is that to obtain a global $L^{\infty}$ bound for the vorticity we need first to establish an $L^{p}$ estimate for some $\left.p \in\right] 2, \infty[$ and it is not clear how to get rid of this condition.

(5) Since $\nabla v \in L_{\text {loc }}^{1}\left(\mathbb{R}_{+} ; L^{\infty}\right)$, we can propagate all the higher regularities, both critical (for example $v_{0} \in B_{p, 1}^{1+2 / p}$ with $p<\infty$ ) and subcritical (for example $v_{0} \in H^{s}$, with $s>2$ ).

\section{Notation and preliminaries}

Throughout this paper we will use the following notation.

- For any positive $A$ and $B$ the notation $A \lesssim B$ means that there exists a positive constant $C$ such that $A \leq C B$.

- For any tempered distribution $u$, both $\hat{u}$ and $\mathscr{F} u$ denote the Fourier transform of $u$.

- For every $p \in[1, \infty]$, we denote by $\|\cdot\|_{L^{p}}$ the norm in the Lebesgue space $L^{p}$.

- The norm in the mixed space time Lebesgue space $L^{p}\left([0, T], L^{r}\left(\mathbb{R}^{d}\right)\right)$ is denoted by $\|\cdot\|_{L_{T}^{p} L^{r}}$ (with the obvious generalization to $\|\cdot\|_{L_{T}^{p} \mathscr{X}}$ for any normed space $\left.\mathscr{X}\right)$.

- For any pair of operators $P$ and $Q$ on some Banach space $\mathscr{X}$, the commutator $[P, Q]$ is given by $P Q-Q P$.

- For $p \in[1, \infty]$, we denote by $\dot{W}^{1, p}$ the space of distributions $u$ such that $\nabla u \in L^{p}$.

Functional spaces. We introduce the so-called Littlewood-Paley decomposition and the corresponding cut-off operators. There exists radial positive functions $\chi \in \mathscr{D}\left(\mathbb{R}^{d}\right)$ and $\varphi \in \mathscr{D}\left(\mathbb{R}^{d} \backslash\{0\}\right)$ such that:

(i) $\chi(\xi)+\sum_{q \geq 0} \varphi\left(2^{-q} \xi\right)=1$ and $\operatorname{supp} \chi \cap \operatorname{supp} \varphi\left(2^{-q}\right)=\varnothing$ for all $q \geq 1$.

(ii) $\operatorname{supp} \varphi\left(2^{-j} \cdot\right) \cap \operatorname{supp} \varphi\left(2^{-k} \cdot\right)=\varnothing$ if $|j-k| \geq 2$.

For every $v \in \mathscr{S}^{\prime}\left(\mathbb{R}^{d}\right)$ we set

$$
\Delta_{-1} v=\chi(D) v, \quad \Delta_{q} v=\varphi\left(2^{-q} D\right) v \text { for } q \in \mathbb{N}, \quad S_{q}=\sum_{j=-1}^{q-1} \Delta_{j} \text { for } q \in \mathbb{N} .
$$


The homogeneous operators are defined by

$$
\dot{\Delta}_{q} v=\varphi\left(2^{-q} \mathrm{D}\right) v \quad \text { and } \quad \dot{S}_{q}=\sum_{j \leq q-1} \dot{\Delta}_{j} \quad \text { for } q \in \mathbb{Z}
$$

From [Bony 1981] we split the product $u v$ into three parts:

$$
u v=T_{u} v+T_{v} u+R(u, v)
$$

with

$$
T_{u} v=\sum_{q} S_{q-1} u \Delta_{q} v \quad \text { and } \quad R(u, v)=\sum_{q} \Delta_{q} u \tilde{\Delta}_{q} v, \quad \text { where } \tilde{\Delta}_{q}=\sum_{i=-1}^{1} \Delta_{q+i}
$$

For $(p, r) \in[1,+\infty]^{2}$ and $s \in \mathbb{R}$ we define the inhomogeneous Besov space $B_{p, r}^{s}$ as the set of tempered distributions $u$ such that

$$
\|u\|_{B_{p, r}^{s}}:=\left(2^{q s}\left\|\Delta_{q} u\right\|_{L^{p}}\right)_{\ell^{r}}<+\infty .
$$

The homogeneous Besov space $\dot{B}_{p, r}^{s}$ is defined as the set of $u \in \mathscr{Y}^{\prime}\left(\mathbb{R}^{d}\right)$ up to polynomials such that

$$
\|u\|_{\dot{B}_{p, r}^{s}}:=\left(2^{q s}\left\|\dot{\Delta}_{q} u\right\|_{L^{p}}\right)_{\ell^{r}(\mathbb{Z})}<+\infty
$$

For $s, s^{\prime} \in \mathbb{R}$ and $p, r \in[1, \infty]$ we define the generalized Besov space $B_{p, r}^{s, s^{\prime}}$ as the set of tempered distributions $u$ such that

$$
\|u\|_{B_{p, r}^{s, s^{\prime}}}:=\left(2^{q s}(|q|+1)^{s^{\prime}}\left\|\Delta_{q} u\right\|_{L^{p}}\right)_{\ell^{r}}<\infty .
$$

Let $T>0$ and $\rho \geq 1$. We denote by $L_{T}^{\rho} B_{p, r}^{s, s^{\prime}}$ the space of distributions $u$ such that

$$
\|u\|_{L_{T}^{\rho} B_{p, r}^{s, s^{\prime}}}:=\left\|\left(2^{q s}(|q|+1)^{s^{\prime}}\left\|\Delta_{q} u\right\|_{L^{p}}\right)_{\ell^{r}}\right\|_{L_{T}^{\rho}}<+\infty
$$

We say that $u$ belongs to the space $\widetilde{L}_{T}^{\rho} B_{p, r}^{s, s^{\prime}}$ if

$$
\|u\|_{\widetilde{L}_{T}^{\rho} B_{p, r}^{s}}:=\left(2^{q s}(|q|+1)^{s^{\prime}}\left\|\Delta_{q} u\right\|_{L_{T}^{\rho} L^{p}}\right)^{r}<+\infty .
$$

By a direct application of the Minkowski inequality, we have the following links between these spaces, for $\varepsilon>0$ :

$$
\begin{aligned}
& L_{T}^{\rho} B_{p, r}^{s} \hookrightarrow \widetilde{L}_{T}^{\rho} B_{p, r}^{s} \hookrightarrow L_{T}^{\rho} B_{p, r}^{s-\varepsilon}, \quad \text { if } r \geq \rho, \\
& L_{T}^{\rho} B_{p, r}^{s+\varepsilon} \hookrightarrow \widetilde{L}_{T}^{\rho} B_{p, r}^{s} \hookrightarrow L_{T}^{\rho} B_{p, r}^{s}, \quad \text { if } \rho \geq r \text {. }
\end{aligned}
$$

We will make frequent use of Bernstein inequalities (see [Chemin 1998], for instance).

Lemma 2.1. There exists a constant $C$ such that, for $q, k \in \mathbb{N}, 1 \leq a \leq b$ and $f \in L^{a}\left(\mathbb{R}^{d}\right)$, we have

and

$$
\sup _{|\alpha|=k}\left\|\partial^{\alpha} S_{q} f\right\|_{L^{b}} \leq C^{k} 2^{q(k+d(1 / a-1 / b))}\left\|S_{q} f\right\|_{L^{a}}
$$

$$
C^{-k} 2^{q k}\left\|\Delta_{q} f\right\|_{L^{a}} \leq \sup _{|\alpha|=k}\left\|\partial^{\alpha} \Delta_{q} f\right\|_{L^{a}} \leq C^{k} 2^{q k}\left\|\Delta_{q} f\right\|_{L^{a}}
$$




\section{Maximum principle}

Our task is to establish some useful estimates for the following equation generalizing (1):

$$
\left\{\begin{array}{l}
\partial_{t} \theta+v \cdot \nabla \theta+\frac{|\mathrm{D}|^{\beta}}{\operatorname{div} v=0} \\
\theta_{\mid t=0}=\theta_{0} .
\end{array}\right.
$$

Two special problems will be studied. One deals with $L^{p}$ estimates that give in particular Theorem 1.1. The second consists in establishing some logarithmic estimates in Besov spaces with index regularity 0 .

The first main result of this section generalizes Theorem 1.1:

Theorem 3.1. Let $p \in[1, \infty], \beta \in] 0,1], \alpha \geq 0$ and $\lambda \geq e^{(3+2 \alpha) / \beta}$. Any smooth solution of (6) satisfies

$$
\|\theta(t)\|_{L^{p}} \leq\left\|\theta_{0}\right\|_{L^{p}}+\int_{0}^{t}\|f(\tau)\|_{L^{p}} d \tau .
$$

The proof is in two steps. The first is to check the result for the free fractional heat equation. More precisely, we will establish that the semigroup $e^{t \mathscr{L}}$, with

$$
\mathscr{L}:=\frac{|\mathrm{D}|^{\beta}}{\log ^{\alpha}(\lambda+|\mathrm{D}|)},
$$

is a contraction in Lebesgue spaces $L^{p}$, for every $p \in[1, \infty[$ of course under suitable conditions on the parameters $\alpha, \beta, \lambda$. This problem is reduced to showing that $\left\|K_{t}\right\|_{L^{1}} \leq 1$. This is equivalent to $K_{t} \in L^{1}$ and $K_{t} \geq 0$. As we will see, to get the integrability of the kernel we do not need any restriction on the value of our parameters. Nevertheless, the positivity of $K_{t}$ requires some restrictions, which are detailed in Theorem 3.1. The second step is to establish the $L^{p}$ estimate for the system (6) and for this purpose we use some results about Lévy operators or, more generally, submarkovian operators.

Positive definite functions. As we will see, there is a strong connection between the positivity of the kernel $K_{t}$ introduced above and the notion of positive definite functions. We will first gather some well-known properties about positive definite functions and recall some useful criteria for characteristic functions. Then, as an application, we will show that the kernel $K_{t}$ is positive under suitable conditions on the parameters involved.

Definition 3.2. Let $f: \mathbb{R}^{d} \rightarrow \mathbb{C}$ be a complex-valued function. We say that $f$ is positive definite if for every integer $n \in \mathbb{N}^{*}$ and every set of points $\left\{x_{j}, j=1, \ldots, n\right\}$ of $\mathbb{R}^{d}$ the matrix $\left(f\left(x_{j}-x_{k}\right)\right)_{1 \leq j, k \leq n}$ is positive Hermitian, that is,

$$
\sum_{j, k=1}^{n} f\left(x_{j}-x_{k}\right) \xi_{j} \bar{\xi}_{k} \geq 0 \text { for all } \xi_{1}, \ldots, \xi_{n} \in \mathbb{C} .
$$

We will give some results about positive definite functions.

(1) From the definition, every positive definite function $f$ satisfies

$$
f(0) \geq 0, \quad f(-x)=\overline{f(x)}, \quad|f(x)| \leq f(0) .
$$


(2) Continuity of a positive definite function $f$ at zero implies continuity everywhere. More precisely,

$$
|f(x)-f(y)| \leq 2 f(0)(f(0)-f(x-y)) .
$$

(3) The sum of two positive definite functions is also positive definite and according to Schur's lemma the product of two positive definite functions is also positive definite and therefore the class of positive definite functions is a convex cone closed under multiplication.

(4) Let $\mu$ be a finite positive measure. Its Fourier-Stieltjes transform is given by

$$
\hat{\mu}(\xi)=\int_{\mathbb{R}^{d}} e^{-i x \cdot \xi} d \mu(x) .
$$

It is easy to see that $\hat{\mu}$ is a positive definite function. Indeed,

$$
\sum_{j, k=1}^{n} \hat{\mu}\left(x_{j}-x_{k}\right) \xi_{j} \bar{\xi}_{k}=\int_{\mathbb{R}^{d}}\left(\sum_{j, k=1}^{n} e^{-i x \cdot x_{j}} \xi_{j} e^{i x \cdot x_{k}} \bar{\xi}_{k}\right) d \mu(x)=\int_{\mathbb{R}^{d}}\left|\sum_{j=1}^{n} e^{-i x \cdot x_{j}} \xi_{j}\right|^{2} d \mu(x) \geq 0 .
$$

The converse of (4) is due to Bochner; see for instance [Bochner 1959, Theorem 19].

Theorem 3.3 (Bochner's theorem). Let $f: \mathbb{R}^{d} \rightarrow \mathbb{C}$ be a continuous positive definite function. Then $f$ is the Fourier transform of a finite positive Borel measure.

There are some criteria for radial functions to be positive definite. For example in dimension one the celebrated criterion of Pólya [1949] states that if $F:[0,+\infty[\rightarrow \mathbb{R}$ is continuous and convex with $F(0)=1$ and $\lim _{r \rightarrow+\infty} F(r)=0$ then $f(x)=F(|x|)$ is positive definite. This criterion was extended to higher dimensions by numerous authors [Askey 1973; Gneiting 2001; Trigub 1989]. We mention only one extension:

Theorem 3.4 (Askey). Let $d \in \mathbb{N}$ and let $F:[0,+\infty[\rightarrow \mathbb{R}$ be a continuous function such that

(1) $F(0)=1$,

(2) the function $r \mapsto(-1)^{d} F^{(d)}(r)$ exists and is convex on $] 0,+\infty[$, and

(3) $\lim _{r \rightarrow+\infty} F(r)=\lim _{r \rightarrow+\infty} F^{(d)}(r)=0$.

Then for every $k \in\{1,2, \ldots, 2 d+1\}$ the function $x \mapsto F(|x|)$ is the Fourier transform of a probability measure on $\mathbb{R}^{k}$.

Remark 3.5. As an application of Askey's theorem we have that $x \mapsto e^{-t|x|^{\beta}}$ is positive definite for all $t>0, \beta \in] 0,1]$ and $d \in \mathbb{N}$. Indeed, the function $F(r)=e^{-t r^{\beta}}$ is completely monotone, that is, $(-1)^{k} F^{(k)}(r) \geq 0$, for all $r>0, k \in \mathbb{N}$. Although the case $\left.\left.\beta \in\right] 1,2\right]$ cannot be reached by this criterion, the result is still true.

The perturbation of the function above by a logarithmic damping is also positive definite:

Proposition 3.6. Let $\alpha, t \in[0,+\infty[\times] 0,+\infty[, \beta \in] 0,1], \lambda \geq e^{(3+2 \alpha) / \beta}$, and define $f: \mathbb{R}^{d} \rightarrow \mathbb{R}$ by

$$
f(x)=e^{-t|x|^{\beta} / \log ^{\alpha}(\lambda+|x|)} \text {. }
$$

Then $f$ is a positive definite function for $d \in\{1,2,3\}$. 
Remarks 3.7. (1) It is possible that this result remains true for higher dimension $d \geq 4$ but we will avoid dealing with this more computational case. We also think that the radial function associated to $f$ is completely monotone.

(2) The lower bound of $\lambda$ is not optimal by our method. In fact we can obtain more precise bounds, but this seems to be irrelevant.

Proof. We write $f(x)=F(|x|)$ with

$$
F(r)=e^{-t \phi(r)} \quad \text { and } \quad \phi(r)=\frac{r^{\beta}}{\log ^{\alpha}(\lambda+r)} .
$$

The function $F$ is smooth on ]0, $\infty$ [ and assumptions (1) and (3) of Theorem 3.4 are satisfied. It follows that the function $f$ is positive definite for $d \in\{1,2,3\}$ if

$$
F^{(3)}(r) \leq 0 \text {. }
$$

Easy computations give for $r>0$,

$$
F^{(3)}(r)=\left[-t \phi^{(3)}(r)+3 t^{2} \phi^{\prime}(r) \phi^{(2)}(r)-t^{3}\left(\phi^{\prime}(r)\right)^{3}\right] F(r) .
$$

We will prove that

$$
\phi^{\prime}(r) \geq 0, \phi^{(2)}(r) \leq 0 \quad \text { and } \quad \phi^{(3)}(r) \geq 0 .
$$

This is sufficient to get $F^{(3)}(r) \leq 0$, for all $r>0$. The first derivative of $\phi$ is given by

$$
\begin{aligned}
\phi^{\prime}(r) & =\frac{\beta r^{\beta-1}}{\log ^{\alpha}(\lambda+r)}-\frac{\alpha r^{\beta}}{(\lambda+r) \log ^{\alpha+1}(\lambda+r)} \\
& =\frac{r^{\beta-1}}{(\lambda+r) \log ^{\alpha+1}(\lambda+r)}(\beta \lambda \log (\lambda+r)+r(\beta \log (\lambda+r)-\alpha)) .
\end{aligned}
$$

We see that if $\lambda$ satisfies

$$
\lambda \geq e^{\alpha / \beta}
$$

then $\phi^{\prime}(r) \geq 0$. For the second derivative of $\phi$ we obtain

$$
\begin{aligned}
\phi^{(2)}(r) & =-\frac{\beta(1-\beta) r^{\beta-2}}{\log ^{\alpha}(\lambda+r)}-\frac{2 \alpha \beta r^{\beta-1}}{(\lambda+r) \log ^{1+\alpha}(\lambda+r)}+\frac{\alpha r^{\beta}}{(\lambda+r)^{2} \log ^{\alpha+1}(\lambda+r)}+\frac{\alpha(\alpha+1) r^{\beta}}{(\lambda+r)^{2} \log ^{\alpha+2}(\lambda+r)} \\
& =\frac{r^{\beta-2}}{\log ^{\alpha}(\lambda+r)}\left[-\beta(1-\beta)-\frac{2 \alpha \beta r}{(\lambda+r) \log (\lambda+r)}+\frac{\alpha r^{2}}{(\lambda+r)^{2} \log (\lambda+r)}+\frac{\alpha(\alpha+1) r^{2}}{(\lambda+r)^{2} \log ^{2}(\lambda+r)}\right] .
\end{aligned}
$$

Since $\left(r^{2}\right) /(\lambda+r)^{2} \leq r /(\lambda+r) \leq 1$, we have

$$
\begin{aligned}
\phi^{(2)}(r) & \leq \frac{r^{\beta-2}}{\log ^{\alpha}(\lambda+r)}\left[(1-\beta)\left(-\beta+\frac{2 \alpha}{\log (\lambda+r)}\right)-\frac{\alpha r}{(\lambda+r) \log (\lambda+r)}\left(1-\frac{\alpha+1}{\log (\lambda+r)}\right)\right] \\
& \leq \frac{r^{\beta-2}}{\log ^{\alpha}(\lambda+r)}\left[(1-\beta)\left(-\beta+\frac{2 \alpha}{\log \lambda}\right)-\frac{\alpha r}{(\lambda+r) \log (\lambda+r)}\left(1-\frac{\alpha+1}{\log \lambda}\right)\right] .
\end{aligned}
$$

Now we choose $\lambda$ such that

$$
-\beta+\frac{2 \alpha}{\log \lambda} \leq 0 \quad \text { and } \quad 1-\frac{\alpha+1}{\log \lambda} \geq 0
$$


which is true whenever

$$
\lambda \geq \max \left(e^{2 \alpha / \beta}, e^{\alpha+1}\right) .
$$

Under this assumption we get

$$
\phi^{(2)}(r) \leq 0 \quad \text { for all } r>0
$$

Similarly we have

$$
\phi^{(3)}(r)=I_{1}+I_{2}+I_{3}+I_{4},
$$

with

$$
\begin{aligned}
& I_{1}=\alpha(\alpha+1) r^{\beta-1} \frac{\log ^{-\alpha-3}(\lambda+r)}{(\lambda+r)^{2}}(3 \lambda \beta \log (\lambda+r)+r(3 \beta \log (\lambda+r)-(2+\alpha))), \\
& I_{2}=\alpha r^{\beta} \frac{\log ^{-2-\alpha}(\lambda+r)}{(\lambda+r)^{3}}\left(-3(1+\alpha)+\left(-3 \beta^{2}+6 \beta-2\right) \log (\lambda+r)\right), \\
& I_{3}=\alpha r^{\beta-2} \frac{\log ^{-1-\alpha}(\lambda+r)}{(\lambda+r)^{3}}\left(\lambda \beta(9-6 \beta) r+3 \lambda^{2} \beta(1-\beta)\right), \\
& I_{4}=(2-\beta)(1-\beta) \beta r^{\beta-3} \log ^{-\alpha}(\lambda+r) .
\end{aligned}
$$

It is easy to see that $I_{3}$ and $I_{4}$ are nonnegative. On the other hand, $I_{1}+I_{2}$ equals

$$
\begin{aligned}
& 3 \lambda \beta \alpha(\alpha+1) r^{\beta-1} \frac{\log ^{-\alpha-2}(\lambda+r)}{(\lambda+r)^{2}} \\
& \quad+\alpha r^{\beta} \frac{\log ^{-2-\alpha}(\lambda+r)}{(\lambda+r)^{3}}\left[-3(1+\alpha)+(\alpha+1)(\lambda+r)\left(3 \beta-\frac{2+\alpha}{\log (\lambda+r)}\right)+\left(-3 \beta^{2}+6 \beta-2\right) \log (\lambda+r)\right] .
\end{aligned}
$$

Since $-3 \beta^{2}+6 \beta-2 \geq-2$ for $\beta \in[0,1]$, and since $-\frac{\log x}{x} \geq-\frac{\log \lambda}{\lambda}$ for all $x \geq \lambda \geq e$, we have

$$
\begin{aligned}
I_{1}+I_{2} & \geq \alpha(\alpha+1) r^{\beta} \frac{\log ^{-2-\alpha}(\lambda+r)}{(\lambda+r)^{3}}\left[-3+(\lambda+r)\left(3 \beta-\frac{2+\alpha}{\log \lambda}-2 \frac{\log (\lambda+r)}{(\alpha+1)(\lambda+r)}\right)\right] \\
& \geq \alpha(\alpha+1) r^{\beta} \frac{\log ^{-2-\alpha}(\lambda+r)}{(\lambda+r)^{2}}\left[3 \beta-\frac{3}{\lambda}-\frac{2+\alpha}{\log \lambda}-\frac{2 \log \lambda}{(\alpha+1) \lambda}\right] .
\end{aligned}
$$

We can check that $\log \lambda \leq \lambda$ and $\log ^{2} \lambda \leq \lambda$, for all $\lambda \geq e$. Thus

$$
\begin{aligned}
I_{1}+I_{2} & \geq \alpha(\alpha+1) r^{\beta} \frac{\log ^{-2-\alpha}(\lambda+r)}{(\lambda+r)^{2}}\left[3 \beta-\frac{1}{\log \lambda}\left(5+\alpha+\frac{2}{\alpha+1}\right)\right] \\
& \geq \alpha(\alpha+1) r^{\beta} \frac{\log ^{-2-\alpha}(\lambda+r)}{(\lambda+r)^{2}}\left[3 \beta-\frac{7+\alpha}{\log \lambda}\right] .
\end{aligned}
$$

We choose $\lambda$ such that

$$
3 \beta-\frac{7+\alpha}{\log \lambda} \geq 0
$$

It follows that $I_{1}+I_{2}$ is nonnegative if

$$
\lambda \geq e^{(7+\alpha) /(3 \beta)} .
$$

A condition that implies this inequality and also (7) and (8) is

$$
\lambda \geq e^{(3+2 \alpha) / \beta} \text {. }
$$


Finally, we get: for all $\alpha \geq 0, \beta \in] 0,1]$, and $\lambda \geq e^{(3+2 \alpha) / \beta}$,

$$
\phi^{(3)}(r) \geq 0 \quad \text { for all } r>0 .
$$

This achieves the proof.

More precise information about the kernel $K_{t}$ is listed now:

Lemma 3.8. Let $\lambda \geq 2$ and denote by $K_{t}$ the element of $\mathscr{Y}^{\prime}\left(\mathbb{R}^{d}\right)$ such that

$$
\widehat{K}_{t}(\xi)=e^{-t|\xi|^{\beta} / \log ^{\alpha}(\lambda+|\xi|)} .
$$

(1) For $(t, \alpha, \beta) \in] 0, \infty[\times \mathbb{R} \times] 0, \infty\left[\right.$ the function $K_{t}$ belongs to $\in L^{1} \cap C_{0}$.

(2) For $d \in\{1,2,3\},(t, \alpha, \beta) \in] 0,+\infty\left[\times[0, \infty[\times] 0,1]\right.$ and $\lambda \geq e^{(3+2 \alpha) / \beta}$, we have

$$
K_{t}(x) \geq 0 \text { for all } x \in \mathbb{R}_{+} \text {and }\left\|K_{t}\right\|_{L^{1}}=1 .
$$

Proof. (1) By definition we have

$$
K_{t}(x)=(2 \pi)^{-d} \int_{\mathbb{R}^{d}} e^{-t|\xi|^{\beta} / \log ^{\alpha}(\lambda+|\xi|)} e^{i x \cdot \xi} d \xi
$$

Let $\mu \geq 0$. Integrating by parts we get

$$
|x|^{\mu} x_{j}^{d} K_{t}(x)=(-2 i \pi)^{-d} \int_{\mathbb{R}^{d}} \partial_{\xi_{j}}^{d}\left(e^{-t\left(\left(|\xi|^{\beta}\right) / \log ^{\alpha}(\lambda+|\xi|)\right)}\right)|x|^{\mu} e^{i x \cdot \xi} d \xi .
$$

On the other hand we have

$$
|x|^{\mu} e^{i x \cdot \xi}=|\mathrm{D}|^{\mu} e^{i x \cdot \xi},
$$

where $|\mathrm{D}|$ is a fractional derivative on the variable $\xi$. Thus we get

$$
|x|^{\mu} x_{j}^{d} K_{t}(x)=(-2 i \pi)^{-d} \int_{\mathbb{R}^{d}}|\mathrm{D}|^{\mu} \partial_{\xi_{j}}^{d}\left(e^{-t|\xi|^{\beta} / \log ^{\alpha}(\lambda+|\xi|)}\right) e^{i x \cdot \xi} d \xi .
$$

Now we use the following representation for $|\mathrm{D}|^{\mu}$ when $\left.\left.\mu \in\right] 0,2\right]$ :

$$
|\mathrm{D}|^{\mu} f(x)=C_{\mu, d} \int_{\mathbb{R}^{d}} \frac{f(x)-f(x-y)}{|y|^{d+\mu}} d y .
$$

It follows that

$$
|x|^{\mu}\left|x_{j}^{d} K_{t}(x)\right| \leq C_{\mu, d} \int_{\mathbb{R}^{2 d}} \frac{\left|\mathscr{K}_{j}(\xi)-\mathscr{K}_{j}(\xi-y)\right|}{|y|^{d+\mu}} d y d \xi
$$

with

$$
\mathscr{K}_{j}(\xi):=\partial_{\xi_{j}}^{d}\left(e^{-t|\xi|^{\beta} / \log ^{\alpha}(\lambda+|\xi|)}\right) .
$$

Now we decompose the integral into two parts:

$$
\begin{aligned}
\int_{\mathbb{R}^{2 d}} \frac{\left|\mathscr{K}_{j}(\xi)-\mathscr{K}_{j}(\xi-y)\right|}{|y|^{d+\mu}} d y d \xi & =\int_{|y| \geq \frac{|\xi|}{2}} \frac{\left|\mathscr{K}_{j}(\xi)-\mathscr{K}_{j}(\xi-y)\right|}{|y|^{d+\mu}} d y d \xi+\int_{|y| \leq \frac{|\xi|}{2}} \frac{\left|\mathscr{K}_{j}(\xi)-\mathscr{K}_{j}(\xi-y)\right|}{|y|^{d+\mu}} d y d \xi \\
& =I_{1}+I_{2} .
\end{aligned}
$$


To estimate the first term we use the following estimate, which can be obtained by straightforward computations:

$$
\left|\mathscr{K}_{j}(\xi)\right| \leq C_{t, \alpha, \beta} \frac{|\xi|^{\beta-d}}{\log ^{\alpha}(\lambda+|\xi|)} e^{-t|\xi|^{\beta} / \log ^{\alpha}(\lambda+|\xi|)} \leq C_{t, \alpha, \beta}|\xi|^{\beta-d} e^{-(t / 2)|\xi|^{\beta} / \log ^{\alpha}(\lambda+|\xi|)}
$$

Hence we get, under the assumption $\mu \in] 0, \beta[$,

$$
\begin{aligned}
I_{1} & \leq C_{t, \alpha, \beta} \int_{|\xi| \leq 2|y|} \frac{1}{|y|^{d+\mu}}\left(|\xi|^{\beta-d} e^{-(t / 2)|\xi|^{\beta} / \log ^{\alpha}(\lambda+|\xi|)}+|\xi-y|^{\beta-d} e^{-(t / 2)|\xi-y|^{\beta} / \log ^{\alpha}(\lambda+|\xi-y|)}\right) d \xi d y \\
& \leq C_{t, \alpha, \beta} \int_{|\xi| \leq 3|y|} \frac{1}{|y|^{d+\mu}}|\xi|^{\beta-d} e^{-(t / 2)|\xi|^{\beta} / \log ^{\alpha}(\lambda+|\xi|)} d \xi d y \leq C_{t, \alpha, \beta} \int_{\mathbb{R}^{d}} \frac{1}{|\xi|^{d+\mu-\beta}} e^{-(t / 2)|\xi|^{\beta} / \log ^{\alpha}(\lambda+|\xi|)} d \xi \\
& \leq C_{t, \alpha, \beta} .
\end{aligned}
$$

To estimate the second term we use the mean-value theorem to write

$$
\left|\mathscr{K}_{j}(\xi)-\mathscr{K}_{j}(\xi-y)\right| \leq|y| \sup _{\eta \in[\xi-y, \xi]}\left|\nabla \mathscr{K}_{j}(\eta)\right|
$$

we also have

$$
\left|\nabla \mathscr{K}_{j}(\eta)\right| \leq C_{t, \alpha, \beta}|\eta|^{\beta-d-1} e^{-(t / 2)|\eta|^{\beta} / \log ^{\alpha}(\lambda+|\eta|)} .
$$

Since $|y| \leq \frac{1}{2}|\xi|$, for $\eta \in[\xi-y, \xi]$ we have

$$
\frac{1}{2}|\xi| \leq|\eta| \leq \frac{5}{2}|\xi|
$$

This yields

$$
\left|\mathscr{K}_{j}(\xi)-\mathscr{K}_{j}(\xi-y)\right| \leq C_{t}|y||\xi|^{\beta-d-1} e^{-C t|\xi|^{\beta / 2}} .
$$

Therefore we find, for $\mu \in] 0, \beta[\cap] 0,1[$,

$$
\begin{aligned}
I_{2} & \leq C_{t, \alpha, \beta} \int_{|y| \leq(1 / 2)|\xi|} \frac{1}{|y|^{d+\mu-1}}|\xi|^{\beta-d-1} e^{-C t|\xi|^{\beta / 2}} d y d \xi \leq C_{t, \alpha, \beta} \int_{\mathbb{R}^{2}}\left(1 /|\xi|^{d+\mu-\beta}\right) e^{-C t|\xi|^{\beta / 2}} d \xi \\
& \leq C_{t, \alpha, \beta} .
\end{aligned}
$$

Finally we get

$$
|x|^{\mu}\left|x_{j}\right|^{d}\left|K_{t}(x)\right| \leq C_{t, \alpha, \beta} \quad \text { for } j=1, \ldots, d .
$$

Since $K_{t} \in C_{0}$, we have

$$
\left(1+|x|^{d+\mu}\right)\left|K_{t}(x)\right| \leq C_{t} .
$$

This proves that $K_{t} \in L^{1}\left(\mathbb{R}^{d}\right)$.

(2) Using Theorem 3.4 and Proposition 3.6 we get $K_{t} \geq 0$. Since $K_{t} \in L^{1}$, this implies $\left\|K_{t}\right\|_{L^{1}}=\widehat{K}_{t}(0)=1$.

Now set

$$
\mathscr{L}:=\frac{|\mathrm{D}|^{\beta}}{\log ^{\alpha}(\lambda+|\mathrm{D}|)} .
$$

We define the propagator $e^{-t \mathscr{L}}$ by convolution:

$$
e^{-t \mathscr{L}} f=K_{t} \star f .
$$


Corollary 3.9. Let $\alpha \geq 0, \beta \in] 0,1], \lambda \geq e^{(3+2 \alpha) / \beta}$ and $p \in[1, \infty]$. Then

$$
\left\|e^{-t \mathscr{L}} f\right\|_{L^{p}} \leq\|f\|_{L^{p}} \quad \text { for all } f \in L^{p} .
$$

Proof. From the classical convolution inequalities combined with Lemma 3.8 we get

$$
\left\|e^{-t \mathscr{L}} f\right\|_{L^{p}} \leq\left\|K_{t}\right\|_{L^{1}}\|f\|_{L^{p}} \leq\|f\|_{L^{p}} .
$$

Structure of the semigroup $\left(\boldsymbol{e}^{-t \mathscr{L}}\right)_{t \geq 0}$. We first recall the notions of $C_{0}$-semigroup and submarkovian generators.

Definition 3.10. Let $X$ be a Banach space and $\left(T_{t}\right)_{t \geq 0}$ a family of bounded operators from $X$ into $X$. This family is called a strongly continuous semigroup on $X$ or a $C_{0}$-semigroup if

(1) $T_{0}=\mathrm{Id}$,

(2) $T_{t+s}=T_{t} T_{s}$ for every $t, s \geq 0$, and

(3) $\lim _{t \rightarrow 0^{+}}\left\|T_{t} x-x\right\|=0$ for every $x \in X$.

If in addition the semigroup satisfies the estimate $\left\|T_{t}\right\|_{\mathscr{L}(X)} \leq 1$, then it is called a $C_{0}$-semigroup of contractions.

For a given $C_{0}$-semigroup of contractions $\left(T_{t}\right)_{t \geq 0}$ we define its domain $\mathscr{D}(A)$ by

$$
\mathscr{D}(A):=\left\{f \in X: \lim _{t \rightarrow 0^{+}} \frac{T_{t} f-f}{t} \text { exists in } X\right\}
$$

and we set

$$
A f=\lim _{t \rightarrow 0^{+}} \frac{T_{t} f-f}{t} \text { for } f \in \mathscr{D}(A) .
$$

It is well-known that the operator $A$ is densely defined: its domain $\mathscr{D}(A)$ is dense in $X$.

Definition 3.11. Let $X=L^{p}\left(\mathbb{R}^{d}\right)$, with $p \in\left[1, \infty\left[\right.\right.$ and $d \in \mathbb{N}^{*}$. A $C_{0}$-semigroup $\left(T_{t}\right)_{t \geq 0}$ of contractions on $X$ is said a submarkovian semigroup if it satisfies these two conditions:

(1) If $f \in X$ satisfies $f(x) \geq 0$ a.e., then $T_{t} f(x) \geq 0$ a.e. for every $t \geq 0$.

(2) If $f \in X$ satisfies $|f| \leq 1$, then $\left|T_{t} f\right| \leq 1$ for every $t \geq 0$.

Define $L_{+}^{p}:=\left\{f \in L^{p} ; f(x) \geq 0\right.$, a.e. $\}$. The next result is classical.

Theorem 3.12 (Beurling-Deny theorem). Let A be a nonnegative self-adjoint operator of $L^{2}$. Then we have equivalence between:

(1) $f \in L_{+}^{2} \Rightarrow e^{-t A} f \in L_{+}^{2}$ for all $t>0$.

(2) $f \in \mathscr{D}\left(A^{1 / 2}\right) \Rightarrow|f| \in \mathscr{D}\left(A^{1 / 2}\right)$ and $\left\|A^{1 / 2}|f|\right\|_{L^{2}} \leq\left\|A^{1 / 2} f\right\|_{L^{2}}$.

Proposition 3.13. Let $d \in\{1,2,3\}, p \in[1, \infty[, \alpha \geq 0, \beta \in] 0,1]$ and $\lambda \geq e^{(3+2 \alpha) / \beta}$. With $\mathscr{L}$ as in (9), we have:

(1) The family $\left(e^{-t \mathscr{L}}\right)_{t \geq 0}$ defines a $C_{0}$-semigroup of contractions in $L^{p}\left(\mathbb{R}^{d}\right)$.

(2) The family $\left(e^{-t \mathscr{L}}\right)_{t \geq 0}$ defines a submarkovian semigroup in $L^{p}\left(\mathbb{R}^{d}\right)$.

(3) The operator $\left(e^{-t \mathscr{L}}\right)_{t \geq 0}$ satisfies the Beurling-Deny theorem described in Theorem 3.12. 
Proof. (1) For $f \in L^{p}$ we define the action of the semigroup on $f$

$$
e^{-t \mathscr{L}} f(x)=K_{t} \star f(x),
$$

where $\widehat{K}_{t}(\xi)=e^{-t|\xi| / \log ^{\alpha}(\lambda+|\xi|)}$. From Corollary 3.9, the semigroup maps $L^{p}$ to itself if $p \in[1, \infty]$, and

$$
\left\|K_{t} \star f\right\|_{L^{p}} \leq\|f\|_{L^{p}} .
$$

Conditions (1) and (2) of Definition 3.10 are easy to check. It remains to prove condition (3), concerning the strong continuity of the semigroup. Since $\left\|K_{t}\right\|_{L^{1}}=1$ and $K_{t} \geq 0$, we have, for $\eta>0$,

$$
\begin{aligned}
K_{t} \star f(x)-f(x) & =\int_{\mathbb{R}^{d}} K_{t}(y)(f(x-y)-f(x)) d y \\
& =\int_{|y| \leq \eta} K_{t}(y)(f(x-y)-f(x)) d y+\int_{|y| \geq \eta} K_{t}(y)(f(x-y)-f(x)) d y \\
& =I_{1}(x)+I_{2}(x) .
\end{aligned}
$$

The first term is estimated as follows:

$$
\left\|I_{1}\right\|_{L^{p}} \leq \int_{|y| \leq \eta} K_{t}(y)\|f(\cdot-y)-f(\cdot)\|_{L^{p}} d y \leq \sup _{|y| \leq \eta}\|f(\cdot-y)-f(\cdot)\|_{L^{p}} .
$$

For the second term we write $\left\|I_{2}\right\|_{L^{p}} \leq 2\|f\|_{L^{p}} \int_{|y| \geq \eta} K_{t}(y) d y$. Combining these estimates we get

$$
\left\|K_{t} \star f-f\right\|_{L^{p}} \leq \sup _{|y| \leq \eta}\|f(\cdot-y)-f(\cdot)\|_{L^{p}}+2\|f\|_{L^{p}} \int_{|y| \geq \eta} K_{t}(y) d y .
$$

It is well known that for every $p \in[1, \infty[$ we have

$$
\lim _{\eta \rightarrow 0^{+}} \sup _{|y| \leq \eta}\|f(\cdot-y)-f(\cdot)\|_{L^{p}}=0 .
$$

Thus for a given $\varepsilon>0$ we can find $\eta>0$ small enough that

$$
\sup _{|y| \leq \eta}\|f(\cdot-y)-f(\cdot)\|_{L^{p}} \leq \varepsilon .
$$

Now to conclude the proof it suffices to prove that

$$
\lim _{t \rightarrow 0^{+}} \int_{|y| \geq \eta} K_{t}(y) d y=0 .
$$

This will follow from

$$
K_{t} \stackrel{t \rightarrow 0^{+}}{\rightarrow} \delta_{0} .
$$

To prove this last statement we write, for $\phi \in \mathscr{Y}$,

$$
\left\langle K_{t}, \phi\right\rangle=\frac{1}{(2 \pi)^{d}}\left\langle\widehat{K}_{t}, \hat{\phi}\right\rangle=\frac{1}{(2 \pi)^{d}} \int_{\mathbb{R}^{d}} e^{-t|\xi|^{\alpha}} \hat{\phi}(\xi) d \xi .
$$

We can use now Lebesgue theorem and the inversion Fourier transform leading to

$$
\lim _{t \rightarrow 0^{+}}\left\langle K_{t}, \phi\right\rangle=\frac{1}{(2 \pi)^{d}} \int_{\mathbb{R}^{d}} \hat{\phi}(\xi) d \xi=\phi(0) .
$$


Finally we get that $\left(K_{t} \star\right)_{t \geq 0}$ defines a $C_{0}$-semigroup of contractions for every $p \in[1, \infty[$.

(2) From Definition 3.11 and the first part of Proposition 3.13 it remains to show that

- for $f \in L^{p}$ with $f(x) \geq 0$ a.e. we have $e^{-t \mathscr{L}} f(x) \geq 0$;

- for $f \in L^{p}$ with $|f(x)| \leq 1$ a.e. we have $\left|e^{-t \mathscr{L}} f(x)\right| \leq 1$.

This is a direct consequence of the explicit formula

$$
e^{-t \mathscr{L}} f(x)=K_{t} \star f(x),
$$

where according to Lemma 3.8 we have $K_{t} \geq 0$ and $\left\|K_{t}\right\|_{L^{1}}=1$.

(3) It is not hard to see that the operator $|\mathrm{D}|^{\beta} / \log ^{\alpha}(\lambda+|\mathrm{D}|)$ is a nonnegative self-adjoint operator of $L^{2}$. This operator satisfies the first condition of Theorem 3.12 since the kernel $K_{t}$ is positive.

The following result gives in particular Theorem 3.1.

Proposition 3.14. Let $A$ be a generator of a $C_{0}$-semigroup of contractions.

(1) Let $p \in[1, \infty[$ and $u \in \mathscr{D}(A)$. Then

$$
\int_{\mathbb{R}^{2}} A u|u|^{p-1} \operatorname{sign} u d x \leq 0 .
$$

(2) Let $\theta$ be a smooth solution of the equation

$$
\partial_{t} \theta+v \cdot \nabla \theta-A \theta=f
$$

where $v$ is a smooth vector field with zero divergence and $f$ is a smooth function. Then

$$
\|\theta(t)\|_{L^{p}} \leq\left\|\theta_{0}\right\|_{L^{p}}+\int_{0}^{t}\|f(\tau)\|_{L^{p}} d \tau \quad \text { for every } p \in[1, \infty] .
$$

Proof. (1) We introduce the operation $[h, g]$ between two functions by

$$
[h, g]=\|g\|_{L^{p}}^{2-p} \int_{\mathbb{R}^{2}} h(x)|g(x)|^{p-1} \operatorname{sign} g(x) d x .
$$

Define the function $\psi:[0, \infty[\rightarrow \mathbb{R}$ by

$$
\psi(t)=\left[e^{t A} u, u\right] .
$$

We have $\psi(0)=\|u\|_{L^{p}}^{2}$. From the Hölder inequality and the fact that the operator $e^{t A}$ is a contraction on $L^{p}$ we get

$$
\psi(t) \leq\left\|e^{t A} u\right\|_{L^{p}}\|u\|_{L^{p}} \leq\|u\|_{L^{p}}^{2} .
$$

Thus we find $\psi(t) \leq \psi(0)$, for all $t \geq 0$. Therefore we get $\lim _{t \rightarrow 0^{+}} \frac{\psi(t)-\psi(0)}{t} \leq 0$. This gives

$$
\int_{\mathbb{R}^{2}} A u(x)|u(x)|^{p-1} \operatorname{sign} u(x) d x \leq 0 .
$$


(2) Let $p \in\left[1, \infty\left[\right.\right.$. Multiplying the first equation in (6) by $|\theta|^{p-1} \operatorname{sign} \theta$, integrating by parts and using $\operatorname{div} v=0$ we get

Using Proposition 3.14 we find

$$
\frac{1}{p} \frac{d}{d t}\|\theta(t)\|_{L^{p}}^{p}+\int_{\mathbb{R}^{2}}|A \theta(x) \theta(x)|^{p-1} \operatorname{sign} \theta(x) d x \leq\|f(t)\|_{L^{p}}\|\theta(t)\|_{L^{p}}^{p-1} .
$$

$$
\frac{1}{p} \frac{d}{d t}\|\theta(t)\|_{L^{p}}^{p} \leq\|f(t)\|_{L^{p}}\|\theta(t)\|_{L^{p}}^{p-1} .
$$

Simplifying, we get $\frac{d}{d t}\|\theta(t)\|_{L^{p}} \leq\|f(t)\|_{L^{p}}$. Integrating in time we get for $p \in[1, \infty[$

$$
\|\theta(t)\|_{L^{p}} \leq\left\|\theta_{0}\right\|_{L^{p}} .
$$

Since the estimates are uniform on the parameter $p$, we can get the limit case $p=+\infty$.

Logarithmic estimate. In the last part of this section we show some logarithmic estimates generalizing results in [Vishik 1998; Hmidi and Keraani 2009]. We recall the following result on the propagation of Besov regularities.

Proposition 3.15. Let $\kappa \geq 0$ and let $A$ be a $C_{0}$ semigroup of contractions on $L^{m}\left(\mathbb{R}^{d}\right)$ for every $m \in[1, \infty[$. We assume that for every $q \in \mathbb{N} \cup\{-1\}$, the operator $\Delta_{q}$ commutes with $A$ on a dense subset of $L^{p}$. Let $\left.(p, r) \in[1, \infty]^{2}, s \in\right]-1,1[$, and let $\theta$ be a smooth solution of

$$
\partial_{t} \theta+v \cdot \nabla \theta-\kappa A \theta=f .
$$

Then

$$
\|\theta\|_{\tilde{L}_{t}^{\infty} B_{p, r}^{s}} \lesssim e^{C V(t)}\left(\left\|\theta_{0}\right\|_{B_{p, r}^{s}}+\int_{0}^{t} e^{-C V(\tau)}\|f(\tau)\|_{B_{p, r}^{s}} d \tau\right),
$$

where $V(t)=\|\nabla v\|_{L_{t}^{1} L^{\infty}}$ and $C$ is a constant depending only on $s$ and $d$.

Proof. We set $\theta_{q}:=\Delta_{q} \theta$. By localizing in frequency the equation of $\theta$ we get

$$
\partial_{t} \theta_{q}+v \cdot \nabla \theta_{q}-\kappa A \theta_{q}=-\left[\Delta_{q}, v \cdot \nabla\right] \theta+f_{q} .
$$

Using Proposition 3.14 we get

$$
\left\|\theta_{q}(t)\right\|_{L^{p}} \leq\left\|\theta_{q}(0)\right\|_{L^{p}}+\int_{0}^{t}\left\|\left[\Delta_{q}, v \cdot \nabla\right] \theta(\tau)\right\|_{L^{p}} d \tau+\int_{0}^{t}\left\|f_{q}(\tau)\right\|_{L^{p}} d \tau .
$$

At the same time, we have the classical commutator estimate [Chemin 1998]:

$$
\left\|\left[\Delta_{q}, v \cdot \nabla\right] \theta\right\|_{L^{p}} \leq C 2^{-q s} c_{q}\|\nabla v\|_{L^{\infty}}\|\theta\|_{B_{p, r}^{s}}, \quad\left\|\left(c_{q}\right)\right\|_{\ell^{r}}=1 .
$$

Thus

$$
\|\theta(t)\|_{B_{p, r}^{s}} \leq\left\|\theta_{0}\right\|_{B_{p, r}^{s}}+C \int_{0}^{t}\|\nabla v\|_{L^{\infty}}\|\theta\|_{B_{p, r}^{s}}+\int_{0}^{t}\|f(\tau)\|_{B_{p, r}^{s}} d \tau .
$$

It suffices now to use the Gronwall's inequality.

Now we will show that for the index regularity $s=0$ we can obtain a better estimate with a linear growth on the norm of the velocity. 
Proposition 3.16. Let $v$ be a smooth divergence-free vector field on $\mathbb{R}^{d}$. Let $\kappa \geq 0$ and let $A$ be $a$ generator of $C_{0}$-semigroup of contractions on $L^{p}\left(\mathbb{R}^{d}\right)$ for every $p \in[1, \infty[$. We assume that for every $q \in \mathbb{N}$, the operators $\Delta_{q}$ and A commute on a dense subset of $L^{p}$. Let $\theta$ be a smooth solution of

$$
\partial_{t} \theta+v \cdot \nabla \theta-\kappa A \theta=f .
$$

Then, for every $p \in[1, \infty]$,

$$
\|\theta\|_{\tilde{L}_{t}^{\infty} B_{p, 1}^{0}} \leq C\left(\left\|\theta_{0}\right\|_{B_{p, 1}^{0}}+\|f\|_{L_{t}^{1} B_{p, 1}^{0}}\right)\left(1+\int_{0}^{t}\|\nabla v(\tau)\|_{L^{\infty}} d \tau\right),
$$

where the constant $C$ does not depend on $p$ or $\kappa$.

This was first proved in [Vishik 1998] for the case $\kappa=0$ by using the special structure of the transport equation. In [Hmidi and Keraani 2008] we generalized Vishik's result for a transport-diffusion equation where the dissipation term has the form $-\kappa \Delta \theta$. The method described in there can be easily adapted here for our model.

Proof. Let $q \in \mathbb{N} \cup\{-1\}$ and denote by $\bar{\theta}_{q}$ the unique global solution of the initial value problem

$$
\left\{\begin{array}{l}
\partial_{t} \bar{\theta}_{q}+v \cdot \nabla \bar{\theta}_{q}-\kappa A \bar{\theta}_{q}=\Delta_{q} f, \\
\bar{\theta}_{q_{\mid t=0}}=\Delta_{q} \theta^{0} .
\end{array}\right.
$$

Using Proposition 3.15 with $s= \pm \frac{1}{2}$ we get

$$
\left\|\bar{\theta}_{q}\right\|_{\widetilde{L}_{t}^{\infty} B_{p, \infty}^{ \pm(1 / 2)}} \lesssim\left(\left\|\Delta_{q} \theta_{0}\right\|_{B_{p, \infty}^{ \pm(1 / 2)}}+\left\|\Delta_{q} f\right\|_{\left.L_{t}^{1} B_{p, \infty}^{ \pm(1 / 2)}\right)} e^{C V(t)},\right.
$$

where $V(t)=\|\nabla v\|_{L_{t}^{1} L^{\infty}}$. Combining this with the definition of Besov spaces this yields, for $j, q \geq-1$,

$$
\left\|\Delta_{j} \bar{\theta}_{q}\right\|_{L_{t}^{\infty} L^{p}} \lesssim 2^{-\frac{1}{2}|j-q|}\left(\left\|\Delta_{q} \theta_{0}\right\|_{L^{p}}+\left\|\Delta_{q} f\right\|_{L_{t}^{1} L^{p}}\right) e^{C V(t)} .
$$

By linearity and again the definition of Besov spaces we have

$$
\|\theta\|_{\tilde{L}_{t}^{\infty} B_{p, 1}^{0}} \leq \sum_{|j-q| \geq N}\left\|\Delta_{j} \bar{\theta}_{q}\right\|_{L_{t}^{\infty} L^{p}}+\sum_{|j-q|<N}\left\|\Delta_{j} \bar{\theta}_{q}\right\|_{L_{t}^{\infty} L^{p}}
$$

where $N \in \mathbb{N}$ is to be chosen later. To deal with the first sum we use (11):

$\sum_{|j-q| \geq N}\left\|\Delta_{j} \bar{\theta}_{q}\right\|_{L_{t}^{\infty} L^{p}} \lesssim 2^{-N / 2} \sum_{q \geq-1}\left(\left\|\Delta_{q} \theta_{0}\right\|_{L^{p}}+\left\|\Delta_{q} f\right\|_{L_{t}^{1} L^{p}}\right) e^{C V(t)} \lesssim 2^{-N / 2}\left(\left\|\theta^{0}\right\|_{B_{p, 1}^{0}}+\|f\|_{L_{t}^{1} B_{p, 1}^{0}}\right) e^{C V(t)}$.

We now turn to the second sum in the right-hand side of (12). It is clear that

$$
\sum_{|j-q|<N}\left\|\Delta_{j} \bar{\theta}_{q}\right\|_{L_{t}^{\infty} L^{p}} \lesssim \sum_{|j-q|<N}\left\|\bar{\theta}_{q}\right\|_{L_{t}^{\infty} L^{p}}
$$

Applying Proposition 3.14 to the system (10) yields $\left\|\bar{\theta}_{q}\right\|_{L_{t}^{\infty} L^{p}} \leq\left\|\Delta_{q} \theta_{0}\right\|_{L^{p}}+\left\|\Delta_{q} f\right\|_{L_{t}^{1} L^{p}}$. It follows that

$$
\sum_{|j-q|<N}\left\|\Delta_{j} \bar{\theta}_{q}\right\|_{L_{t}^{\infty} L^{p}} \lesssim N\left(\left\|\theta^{0}\right\|_{B_{p, 1}^{0}}+\|f\|_{L_{t}^{1} B_{p, 1}^{0}}\right) .
$$

The outcome is that

$$
\|\theta\|_{\tilde{L}_{t}^{\infty} B_{p, 1}^{0}} \lesssim\left(\left\|\theta^{0}\right\|_{B_{p, 1}^{0}}+\|f\|_{L_{t}^{1} B_{p, 1}^{0}}\right)\left(2^{-N / 2} e^{C V(t)}+N\right)
$$


Choosing

$$
N=\left[\frac{2 C V(t)}{\log 2}\right]+1
$$

we get the desired result.

Combining Propositions 3.16 and 3.13 we get:

Corollary 3.17. Let $v$ be a smooth divergence-free vector field on $\mathbb{R}^{d}$, with $d \in\{2,3\}$. Let $\kappa, \alpha \geq 0$, $\beta \in] 0,1], \lambda \geq e^{(3+2 \alpha) / \beta}$, and $p \in[1, \infty]$. Let $\theta$ be a smooth solution of

$$
\partial_{t} \theta+v \cdot \nabla \theta+\kappa|\mathrm{D}|^{\beta} \log ^{-\alpha}(\lambda+|\mathrm{D}|) \theta=f .
$$

Then

$$
\|\theta\|_{\widetilde{L}_{t}^{\infty} B_{p, 1}^{0}} \leq C\left(\left\|\theta_{0}\right\|_{B_{p, 1}^{0}}+\|f\|_{L_{t}^{1} B_{p, 1}^{0}}\right)\left(1+\int_{0}^{t}\|\nabla v(\tau)\|_{L^{\infty}} d \tau\right),
$$

where the constant $C$ depends only on $\lambda$ and $\alpha$.

\section{Proof of the generalized Bernstein inequality (Theorem 1.3)}

We first extend the classical Bernstein inequality of Lemma 2.1 to more general operators:

Proposition 4.1. Let $\alpha \in \mathbb{R}, \beta>0$ and $\lambda \geq 2$. Then there exists a constant $C$ such that for every $f \in \mathscr{T}\left(\mathbb{R}^{d}\right)$ and for every $q \geq-1$ and $p \in[1, \infty]$ we have

$$
\left\|\Delta_{q}(\mathscr{L} f)\right\|_{L^{p}} \leq C 2^{q \beta}(|q|+1)^{-\alpha}\left\|\Delta_{q} f\right\|_{L^{p}}
$$

where $\mathscr{L}=\frac{|\mathrm{D}|^{\beta}}{\log ^{\alpha}(\lambda+|\mathrm{D}|)}($ as in (9)). Moreover

$$
\left\|S_{q}(\mathscr{L} f)\right\|_{L^{p}} \leq C 2^{q \beta}(|q|+1)^{-\alpha}\left\|S_{q} f\right\|_{L^{p}} .
$$

Remark 4.2. The first result of Proposition 4.1 remains true for more general situation where $q \in \mathbb{N}$ and the operator $|\mathrm{D}|^{\beta}$ is replaced by $a(\mathrm{D})$, where $a \in C^{\infty}(\mathbb{R} \backslash\{0\})$ is a homogeneous distribution of order $\beta \in \mathbb{R}$ satisfying

$$
\left|\partial_{\xi}^{\gamma} a(\xi)\right| \leq C|\xi|^{\beta-|\gamma|} \quad \text { for every } \gamma \in \mathbb{N}^{d} .
$$

Proof of Proposition 4.1. Case $q \in \mathbb{N}$. It is easy to see that

$$
\Delta_{q}\left(\frac{|\mathrm{D}|^{\beta}}{\log ^{\alpha}(\lambda+|\mathrm{D}|)} f\right)=K_{q} \star \Delta_{q} f
$$

with

$$
\widehat{K}_{q}(\xi)=\frac{\tilde{\phi}\left(2^{-q} \xi\right)|\xi|^{\beta}}{\log ^{\alpha}(\lambda+|\xi|)}
$$

for $\tilde{\phi}$ a smooth function supported in the annulus $\left\{\frac{1}{4} \leq|x| \leq 3\right\}$ and taking the value 1 on the support of the function $\phi$ introduced in Section 2. By Fourier inversion and change of variables we get

$$
K_{q}(x)=c_{d} \int_{\mathbb{R}^{d}} e^{i x \cdot \xi} \frac{\tilde{\phi}\left(2^{-q} \xi\right)|\xi|^{\beta}}{\log ^{\alpha}(\lambda+|\xi|)} d \xi=c_{d} 2^{q \beta} 2^{q d} \int_{\mathbb{R}^{d}} e^{i 2^{q} x \cdot \xi} \frac{\tilde{\phi}(\xi)|\xi|^{\beta}}{\log ^{\alpha}\left(\lambda+2^{q}|\xi|\right)} d \xi=c_{d} 2^{q \beta} 2^{q d} \tilde{K}_{q}\left(2^{q} x\right)
$$


with

$$
\tilde{K}_{q}(x)=\int_{\mathbb{R}^{d}} e^{i x \cdot \xi} \frac{\tilde{\phi}(\xi)|\xi|^{\beta}}{\log ^{\alpha}\left(\lambda+2^{q}|\xi|\right)} d \xi
$$

Obviously we have

$$
\left\|K_{q}\right\|_{L^{1}}=c_{d} 2^{q \beta}\left\|\tilde{K}_{q}\right\|_{L^{1}} .
$$

Hence to prove Proposition 4.1 it suffices to establish

$$
\left\|\tilde{K}_{q}\right\|_{L^{1}} \leq C(q+1)^{-\alpha} .
$$

From the definition of $\tilde{K}_{q}$ we see that

$$
\tilde{K}_{q}(x)=\int_{\mathbb{R}^{d}} e^{i x \cdot \xi} \frac{\tilde{\psi}(\xi)}{\log ^{\alpha}\left(\lambda+2^{q}|\xi|\right)} d \xi
$$

where $\tilde{\psi}$ belongs to the Schwartz class and is supported in $\left\{\frac{1}{4} \leq|x| \leq 3\right\}$. Integrating by parts we get, for $j \in\{1,2, \ldots, d\}$,

$$
x_{j}^{d+1} \tilde{K}_{q}(x)=(-i)^{d+1} \int_{\frac{1}{4} \leq|\xi| \leq 3} e^{i x \cdot \xi} \partial_{\xi_{j}}^{d+1}\left(\frac{\tilde{\psi}(\xi)}{\log ^{\alpha}\left(\lambda+2^{q}|\xi|\right)}\right) d \xi .
$$

Now we claim that

$$
\left|\partial_{\xi_{j}}^{d+1}\left(\frac{\tilde{\psi}(\xi)}{\log ^{\alpha}\left(\lambda+2^{q}|\xi|\right)}\right)\right| \leq C_{\lambda, \alpha, d} \frac{g(\xi)}{\log ^{\alpha}\left(\lambda+2^{q}\right)},
$$

where $g \in \mathscr{Y}\left(\mathbb{R}^{d}\right)$. This is an easy consequence of Leibniz formula and the fact that

$$
\left|\partial_{\xi_{j}}^{n}\left(\frac{1}{\log ^{\alpha}\left(\lambda+2^{q}|\xi|\right)}\right)\right| \leq \sum_{l, k=1}^{n} c_{l, k}\left(\frac{2^{q}}{\lambda+2^{q}|\xi|}\right)^{l} \frac{1}{\log ^{\alpha+k}\left(\lambda+2^{q}|\xi|\right)} \leq \frac{C_{\lambda, \alpha, n}}{\log ^{\alpha}\left(\lambda+2^{q}\right)} \quad \text { for } \frac{1}{4} \leq|\xi| \leq 2 .
$$

Thus we get for $j \in\{1, \ldots, d\}$

$$
\left|x_{j}\right|^{d+1}\left|\tilde{K}_{q}(x)\right| \leq C \log ^{-\alpha}\left(\lambda+2^{q}\right) \quad \text { for } x \in \mathbb{R}^{d} .
$$

It follows that

$$
|x|^{d+1}\left|\tilde{K}_{q}(x)\right| \leq C \log ^{-\alpha}\left(\lambda+2^{q}\right) \text { for } x \in \mathbb{R}^{d} .
$$

It is easy to see that $\tilde{K}_{q}$ is continuous and

$$
\left|\tilde{K}_{q}(x)\right| \leq C \log ^{-\alpha}\left(\lambda+2^{q}\right)
$$

Consequently,

$$
\left|\tilde{K}_{q}(x)\right| \leq C \log ^{-\alpha}\left(\lambda+2^{q}\right)(1+|x|)^{-d-1} \text { for } x \in \mathbb{R}^{d} .
$$

This yields $\left\|\tilde{K}_{q}\right\|_{L^{1}} \leq C \log ^{-\alpha}\left(\lambda+2^{q}\right) \leq C(q+1)^{-\alpha}$, which concludes the proof when $q \in \mathbb{N}$.

Case $q=-1$. Here we can write the kernel $K_{-1}$ as

$$
K_{-1}(x)=\int_{\mathbb{R}^{d}} e^{i x \cdot \xi} \frac{\tilde{\chi}(\xi)|\xi|^{\beta}}{\log ^{\alpha}(\lambda+|\xi|)} d \xi=\int_{\mathbb{R}^{d}} e^{i x \cdot \xi} \chi_{1}(\xi) d \xi
$$


where $\tilde{\chi}$ is a smooth compactly supported function taking the value 1 on the support of the function $\chi$ introduced in Section 2. The function $\chi_{1}$ is given by

$$
\chi_{1}(\xi)=\frac{\tilde{\chi}(\xi)|\xi|^{\beta}}{\log ^{\alpha}(\lambda+|\xi|)} .
$$

We can see by means of easy computations that $\tilde{\chi}$ is smooth outside zero and satisfies, for every $\gamma \in \mathbb{N}^{d}$,

$$
\left|\partial_{\xi}^{\gamma} \tilde{\chi}(\xi)\right| \leq C_{\gamma}|\xi|^{\beta-|\gamma|} \text { for all } \xi \neq 0
$$

Using the Mikhlin-Hörmander theorem we get

$$
\left|K_{-1}(x)\right| \leq C|x|^{-d-\beta} .
$$

Since $K_{-1}$ is continuous at zero we have

$$
\left|K_{-1}(x)\right| \leq C(1+|x|)^{-d-\beta} .
$$

This proves that $K_{-1} \in L^{1}$.

To prove the second estimate we use the first result combined with the identity $S_{q+2} S_{q}=S_{q}$ :

$$
\left\|S_{q}(\mathscr{L} f)\right\|_{L^{p}} \leq \sum_{j=-1}^{q+1}\left\|\Delta_{j}\left(\mathscr{L} S_{q} f\right)\right\|_{L^{p}} \leq C\left\|S_{q} f\right\|_{L^{p}} \sum_{j=-1}^{q+1} 2^{j \beta}(|j|+1)^{-\alpha} .
$$

Since $\beta>0$, this last series diverges and

$$
\sum_{j=-1}^{q+1} 2^{j \beta}(|j|+1)^{-\alpha} \leq C 2^{q \beta}(|q|+1)^{-\alpha} .
$$

This can be deduced from the asymptotic behavior

$$
\int_{1}^{x} e^{\beta t} t^{-\alpha} d t \approx \frac{1}{\beta} e^{\beta x} x^{-\alpha} \quad \text { as } x \rightarrow+\infty .
$$

As a consequence of Proposition 4.1 we get the following result, which describes the action of the logarithmic Riesz transform

$$
\mathscr{R}_{\alpha}=\frac{\partial_{1} \log ^{\alpha}(\lambda+|\mathrm{D}|)}{|\mathrm{D}|}
$$

on Besov spaces.

Corollary 4.3. Let $\alpha \in \mathbb{R}, \lambda>1$ and $p \in[1, \infty]$. The map

$$
\left(\mathrm{Id}-\Delta_{-1}\right) \mathscr{R}_{\alpha}: B_{p, r}^{s, \alpha} \rightarrow B_{p, r}^{s}
$$

is continuous. 
The generalized Bernstein inequality. In this section we prove Theorem 1.3, which we restate here for convenience:

Theorem 1.3. Let $d \in\{1,2,3\}, \beta \in] 0,1], \alpha \geq 0, \lambda \geq e^{(3+2 \alpha) / \beta}$ and $p>1$. For $q \in \mathbb{N}$ and $f \in \mathscr{S}\left(\mathbb{R}^{d}\right)$,

$$
2^{q \beta}(q+1)^{-\alpha}\left\|\Delta_{q} f\right\|_{L^{p}}^{p} \leq C \int_{\mathbb{R}^{d}}\left(\frac{|\mathrm{D}|^{\beta}}{\log ^{\alpha}(\lambda+|\mathrm{D}|)} \Delta_{q} f\right)\left|\Delta_{q} f\right|^{p-1} \operatorname{sign} \Delta_{q} f d x .
$$

where $C$ depends on $p, \alpha, \beta$ and $\lambda$.

Some preliminary lemmas will be needed. The first is a Stroock-Varopoulos inequality for submarkovian operators. For the proof see [Liskevich et al. 1996; Liskevich and Semenov 1996].

Theorem 4.4. If $p>1$ and $A$ is a submarkovian generator, we have

$$
4 \frac{p-1}{p^{2}}\left\|A^{1 / 2}\left(|f|^{p / 2} \operatorname{sign} f\right)\right\|_{L^{2}}^{2} \leq \int_{\mathbb{R}^{d}}(A f)|f|^{p-1} \operatorname{sign} f d x \leq C_{p}\left\|A^{1 / 2}\left(|f|^{p / 2} \operatorname{sign} f\right)\right\|_{L^{2}}^{2} .
$$

The generator A satisfies the first Beurling-Deny condition

$$
4 \frac{p-1}{p^{2}}\left\|A^{1 / 2}\left(|f|^{p / 2}\right)\right\|_{L^{2}}^{2} \leq \int_{\mathbb{R}^{d}}(A f)|f|^{p-1} \operatorname{sign} f d x .
$$

Combining this result with Proposition 3.13 we get:

Corollary 4.5. Let $p>1, \beta \in] 0,1], \alpha \geq 0$ and $\lambda \geq e^{(3+2 \alpha) / \beta}$. Then

$$
4 \frac{p-1}{p^{2}}\left\|\frac{|\mathrm{D}|^{\beta / 2}}{\log ^{\alpha / 2}(\lambda+|\mathrm{D}|)}\left(|f|^{p / 2}\right)\right\|_{L^{2}}^{2} \leq \int_{\mathbb{R}^{d}}\left(\frac{|\mathrm{D}|^{\beta}}{\log ^{\alpha}(\lambda+|\mathrm{D}|)} f\right)|f|^{p-1} \operatorname{sign} f d x .
$$

We will make use of the following composition results:

Lemma 4.6. (1) Let $\mu \geq 1$ and $s \in[0, \mu[\cap[0,2[$. Then

$$
\left\||f|^{\mu}\right\|_{B_{2,2}^{s}} \leq C\|f\|_{B_{2 \mu, 2}^{s}}\|f\|_{B_{2 \mu, 2}^{0}}^{\mu-1}
$$

(2) Let $\mu \in] 0,1], p, q \in[1, \infty]$ and $0<s<1+\frac{1}{p}$. Then

$$
\left\||f|^{\mu}\right\|_{B_{(p / \mu),(q / \mu)}^{s \mu}} \leq C\|f\|_{B_{p, q}^{s}}^{\mu} .
$$

The first estimate is a particular case of a general result in [Chen et al. 2007]. The second was established in [Sickel 1999]; see also [Kateb 2003, Theorem 1.4].

Next we recall the following result, proved in [Chen et al. 2007; Danchin 2001; Planchon 2000].

Proposition 4.7. Let $d \geq 1, \beta \in] 0,2]$ and $p \geq 2$. Then we have for $q \in \mathbb{N}$ and $f \in \mathscr{S}\left(\mathbb{R}^{d}\right)$,

$$
2^{q \beta}\left\|\Delta_{q} f\right\|_{L^{p}}^{p} \leq C \int_{\mathbb{R}^{d}}\left(|\mathrm{D}|^{\beta} \Delta_{q} f\right)\left|\Delta_{q} f\right|^{p-1} \operatorname{sign} \Delta_{q} f d x .
$$

where $C$ depends on $p$ and $\beta$. For $\beta=2$ we can extend the inequality above to $p \in] 1, \infty[$.

Proof of Theorem 1.3. Using Corollary 4.5 it suffices to prove

$$
C^{-1} 2^{q \beta}(q+1)^{-\alpha}\left\|\Delta_{q} f\right\|_{L^{p}}^{p} \leq\left\|\frac{|\mathrm{D}|^{\beta / 2}}{\log ^{\alpha / 2}(\lambda+|\mathrm{D}|)}\left(\left|\Delta_{q} f\right|^{p / 2}\right)\right\|_{L^{2}}^{2} .
$$


We will use an idea from [Chen et al. 2007]. Let $N \in \mathbb{N}$ then we have

$$
\left\||\mathrm{D}|\left(\left|f_{q}\right|^{p / 2}\right)\right\|_{L^{2}} \leq\left\|S_{N}|\mathrm{D}|\left(\left|f_{q}\right|^{p / 2}\right)\right\|_{L^{2}}+\left\|\left(\mathrm{Id}-S_{N}\right)|\mathrm{D}|\left(\left|f_{q}\right|^{p / 2}\right)\right\|_{L^{2}} .
$$

It is clear that for $s \geq 0$

$$
\left\|\left(\operatorname{Id}-S_{N}\right)|\mathrm{D}|\left(\left|f_{q}\right|^{p / 2}\right)\right\|_{L^{2}} \leq C 2^{-N s}\left\|\left|f_{q}\right|^{p / 2}\right\|_{B_{2,2}^{1+s}} .
$$

We have now to deal with fraction powers in Besov spaces. We will treat differently the cases $p>2$ and $p \leq 2$.

Case $p>2$. Combining Lemma 4.6(1) with the Bernstein inequality we get, under the assumption that $\overline{0<s<\min }(p / 2-1,2)$,

$$
\left\|\left|f_{q}\right|^{p / 2}\right\|_{B_{2,2}^{1+s}} \leq C\left\|f_{q}\right\|_{B_{p, 2}^{0}}^{(p / 2)-1}\left\|f_{q}\right\|_{B_{p, 2}^{1+s}} \leq C 2^{q(1+s)}\left\|f_{q}\right\|_{L^{p}}^{p / 2} .
$$

Case $1<p \leq 2$. Using Lemma 4.6(2) and the Bernstein inequality we get, for $0<s<(p-1) / 2$,

$$
\left\|\left|f_{q}\right|^{p / 2}\right\|_{B_{2,2}^{1+s}} \leq C\left\|f_{q}\right\|_{B_{p, p}^{(2+2 s) / p}}^{p / 2} \leq C 2^{q(1+s)}\left\|f_{q}\right\|_{L^{p}}^{p / 2} .
$$

It follows from (14) and the previous inequalities that there exists $s_{p}>0$ such that for $0<s<s_{p}$

$$
\left\|\left(\mathrm{Id}-S_{N}\right)|\mathrm{D}|\left(\left|f_{q}\right|^{p / 2}\right)\right\|_{L^{2}} \leq C 2^{-N s} 2^{q(1+s)}\left\|f_{q}\right\|_{L^{p}}^{p / 2} .
$$

On the other hand Proposition 4.1 gives

$$
\begin{aligned}
\left\|S_{N}|\mathrm{D}|\left(\left|f_{q}\right|^{p / 2}\right)\right\|_{L^{2}} & \leq\left\|S_{N}|\mathrm{D}|^{1-\beta / 2} \log ^{\alpha / 2}(\lambda+|\mathrm{D}|)\left(\frac{|\mathrm{D}|^{\beta / 2}}{\log ^{\alpha / 2}(\lambda+|\mathrm{D}|)}\left(\left|f_{q}\right|^{p / 2}\right)\right)\right\|_{L^{2}} \\
& \leq C 2^{N(1-\beta / 2)} N^{\alpha / 2}\left\|\frac{|\mathrm{D}|^{\beta / 2}}{\log ^{\alpha / 2}(\lambda+|\mathrm{D}|)}\left(\left|f_{q}\right|^{p / 2}\right)\right\|_{L^{2}} \cdot
\end{aligned}
$$

Therefore we get

$$
\left\||\mathrm{D}|\left(\left|f_{q}\right|^{p / 2}\right)\right\|_{L^{2}} \leq C 2^{-N s} 2^{q(1+s)}\left\|f_{q}\right\|_{L^{p}}^{p / 2}+C 2^{N(1-\beta / 2)} N^{\alpha / 2}\left\|\frac{|\mathrm{D}|^{\beta / 2}}{\log ^{\alpha / 2}(\lambda+|\mathrm{D}|)}\left(\left|f_{q}\right|^{p / 2}\right)\right\|_{L^{2}} .
$$

According to Proposition 4.7 we have for $p \in] 1, \infty[$,

$$
C_{p} 2^{q}\left\|f_{q}\right\|_{L^{p}}^{p / 2} \leq\left\||\mathrm{D}|\left(\left|f_{q}\right|^{p / 2}\right)\right\|_{L^{2}} .
$$

Combining the last two estimates we get

$$
2^{q}\left\|f_{q}\right\|_{L^{p}}^{p / 2} \leq C 2^{s(q-N)} 2^{q}\left\|f_{q}\right\|_{L^{p}}^{p / 2}+C 2^{N(1-\beta / 2)} N^{\alpha / 2}\left\|\frac{|\mathrm{D}|^{\beta / 2}}{\log ^{\alpha / 2}(\lambda+|\mathrm{D}|)}\left(\left|f_{q}\right|^{p / 2}\right)\right\|_{L^{2}} .
$$

We take $N=q+N_{0}$ such that $C 2^{-N_{0} s} \leq \frac{1}{2}$. Then we get

$$
\left\|f_{q}\right\|_{L^{p}}^{p / 2} \leq C 2^{-q \beta / 2}(q+1)^{\alpha / 2}\left\|\frac{|\mathrm{D}|^{\beta / 2}}{\log ^{\alpha / 2}(\lambda+|\mathrm{D}|)}\left(\left|f_{q}\right|^{p / 2}\right)\right\|_{L^{2}} .
$$

This gives the desired result. 


\section{Commutator estimates}

We will establish in this section some commutator estimates. The following result was proved in [Hmidi et al. 2011].

Lemma 5.1. Given $(p, m) \in[1, \infty]^{2}$ such that $p \geq m^{\prime}$ with $m^{\prime}$ the conjugate exponent of $m$. Let $f, g, h$ be functions such that $\nabla f \in L^{p}, g \in L^{m}$ and $x h \in L^{m^{\prime}}$. Then,

$$
\|h \star(f g)-f(h \star g)\|_{L^{p}} \leq\|x h\|_{L^{m^{\prime}}}\|\nabla f\|_{L^{p}}\|g\|_{L^{m}} .
$$

Lemma 5.2. Let $\left(a_{n}\right)_{n \in \mathbb{Z}}$ be a sequence of strictly nonnegative real numbers such that

$$
M:=\max \left(\sup _{n \in \mathbb{Z}} a_{n}^{-1} \sum_{j \leq n} a_{j}, \sup _{n \in \mathbb{Z}} a_{n} \sum_{j \geq n} a_{j}^{-1}\right)<\infty .
$$

For every $p \in[1, \infty]$, the linear operator $T: \ell^{p} \rightarrow \ell^{p}$ defined by

is continuous and $\|T\|_{\mathscr{L}\left(\ell^{p}\right)} \leq M$.

$$
T\left(\left(b_{n}\right)_{n \in \mathbb{Z}}\right)=\left(\sum_{j \leq n} a_{j} a_{n}^{-1} b_{j}\right)_{n \in \mathbb{Z}}
$$

Proof. By interpolation it suffices to prove the cases $p=1$ and $p=+\infty$. Let's start with $p=1$ and set $\boldsymbol{b}=\left(b_{n}\right)_{n \in \mathbb{Z}}$. From the Fubini lemma and the hypothesis we have

$$
\|T \boldsymbol{b}\|_{\ell^{1}} \leq \sum_{n \in \mathbb{Z}} \sum_{j \leq n} a_{j} a_{n}^{-1}\left|b_{j}\right| \leq \sum_{j \in \mathbb{Z}}\left|b_{j}\right| a_{j} \sum_{n \geq j} a_{n}^{-1} \leq M\|\boldsymbol{b}\|_{\ell^{1}} .
$$

For the case $p=+\infty$, we write

$$
\|T \boldsymbol{b}\|_{\ell^{\infty}} \leq \sup _{n \in \mathbb{Z}} \sum_{j \leq n} a_{j} a_{n}^{-1}\left|b_{j}\right| \leq\|\boldsymbol{b}\|_{\ell^{\infty}} \sup _{n \in \mathbb{Z}} a_{n}^{-1} \sum_{j \leq n} a_{j} \leq M\|\boldsymbol{b}\|_{\ell^{\infty}} .
$$

This completes the proof.

The goal now is to study the commutation between the operators

$$
\mathscr{R}_{\alpha}=\frac{\partial_{1}}{|\mathrm{D}|} \log ^{\alpha}(\lambda+|\mathrm{D}|) \quad \text { and } \quad v \cdot \nabla .
$$

Recall that $B_{\infty, 2}^{s, s^{\prime}}$ is the space given by the set of tempered distributions $u$ such that

$$
\|u\|_{B_{\infty, r}^{s, s^{\prime}}}=\left\|\left(2^{q s}(|q|+1)^{s^{\prime}}\left\|\Delta_{q} u\right\|_{L^{\infty}}\right)_{q}\right\|_{\ell^{r}} .
$$

The main result of this section reads as follows.

Proposition 5.3. Let $\alpha \in \mathbb{R}, \lambda>1$, and let $v$ be a smooth divergence-free vector field and $\theta$ a smooth scalar function.

(1) For every $(p, r) \in[2, \infty[\times[1, \infty]$ there exists a constant $C=C(p, r)$ such that

$$
\left\|\left[\mathscr{R}_{\alpha}, v \cdot \nabla\right] \theta\right\|_{B_{p, r}^{0}} \leq C\|\nabla v\|_{L^{p}}\left(\|\theta\|_{B_{\infty, r}^{0, \alpha}}+\|\theta\|_{L^{p}}\right) .
$$

(2) For every $(r, \rho) \in[1, \infty] \times] 1, \infty[$ and $\epsilon>0$ there exists a constant $C=C(r, \rho, \epsilon)$ such that

$$
\left\|\left[\mathscr{R}_{\alpha}, v \cdot \nabla\right] \theta\right\|_{B_{\infty, r}^{0}} \leq C\left(\|\omega\|_{L^{\infty}}+\|\omega\|_{L^{\rho}}\right)\left(\|\theta\|_{B_{\infty, r}^{\epsilon}}+\|\theta\|_{L^{\rho}}\right) .
$$


Proof. (1) We split the commutator into three parts according to Bony's decomposition [1981]:

$$
\begin{aligned}
{\left[\mathscr{R}_{\alpha}, v \cdot \nabla\right] \theta } & =\sum_{q \in \mathbb{N}}\left[\Re_{\alpha}, S_{q-1} v \cdot \nabla\right] \Delta_{q} \theta+\sum_{q \in \mathbb{N}}\left[\Re_{\alpha}, \Delta_{q} v \cdot \nabla\right] S_{q-1} \theta+\sum_{q \geq-1}\left[\Re_{\alpha}, \Delta_{q} v \cdot \nabla\right] \widetilde{\Delta}_{q} \theta \\
& =\sum_{q \in \mathbb{N}} \mathrm{I}_{q}+\sum_{q \in \mathbb{N}} \mathrm{II}_{q}+\sum_{q \geq-1} \mathrm{III}_{q}=\mathrm{I}+\mathrm{II}+\mathrm{III} .
\end{aligned}
$$

We start with the estimate of the term I. It is easy to see that there exists $\tilde{\varphi} \in \mathscr{Y}$ whose spectrum does not meet the origin such that

$$
\mathrm{I}_{q}(x)=h_{q} \star\left(S_{q-1} v \cdot \nabla \Delta_{q} \theta\right)-S_{q-1} v \cdot\left(h_{q} \star \nabla \Delta_{q} \theta\right),
$$

where

$$
\hat{h}_{q}(\xi)=i \tilde{\varphi}\left(2^{-q} \xi\right) \frac{\xi_{1}}{|\xi|} \log ^{\alpha}(\lambda+|\xi|) .
$$

Applying Lemma 5.1 with $m=\infty$ we get

$$
\left\|\mathrm{I}_{q}\right\|_{L^{p}} \lesssim\left\|x h_{q}\right\|_{L^{1}}\left\|\nabla S_{q-1} v\right\|_{L^{p}}\left\|\Delta_{q} \nabla \theta\right\|_{L^{\infty}} \lesssim 2^{q}\left\|x h_{q}\right\|_{L^{1}}\left\|\Delta_{q} \theta\right\|_{L^{\infty}}\|\nabla v\|_{L^{p}} .
$$

We can easily check that

$$
\left\|x h_{q}\right\|_{L^{1}}=2^{-q}\left\|x \tilde{h}_{q}\right\|_{L^{1}} \quad \text { with } \quad \tilde{\tilde{h}}_{q}(\xi)=i \tilde{\varphi}(\xi) \frac{\xi_{1}}{|\xi|} \log ^{\alpha}\left(\lambda+2^{q}|\xi|\right) .
$$

We can get, in a way similar to the proof of Proposition 4.1,

$$
\left\|\tilde{h}_{q}\right\|_{L^{1}} \leq C(1+|q|)^{\alpha} .
$$

Thus estimate (15) becomes

$$
\left\|\mathrm{I}_{q}\right\|_{L^{p}} \leq C(1+|q|)^{\alpha}\left\|\Delta_{q} \theta\right\|_{L^{\infty}}\|\nabla v\|_{L^{p}} .
$$

Combined with the trivial fact

$$
\Delta_{j} \sum_{q} \mathrm{I}_{q}=\sum_{|j-q| \leq 4} \mathrm{I}_{q}
$$

this yields

$$
\|\mathrm{I}\|_{B_{p, r}^{0}} \lesssim\left(\sum_{q \geq-1}\left\|\mathrm{I}_{q}\right\|_{L^{p}}^{r}\right)^{1 / r} \lesssim\|\nabla v\|_{L^{p}}\|\theta\|_{B_{\infty, r}^{0, \alpha}} .
$$

Let us move to the term II. As before we write

$$
\mathrm{II}_{q}(x)=h_{q} \star\left(\Delta_{q} v \cdot \nabla S_{q-1} \theta\right)-\Delta_{q} v \cdot\left(h_{q} \star \nabla S_{q-1} \theta\right),
$$

and then we obtain the estimate

$$
\left\|\mathrm{II}_{q}\right\|_{L^{p}} \lesssim 2^{-q}(1+|q|)^{\alpha}\left\|\Delta_{q} \nabla v\right\|_{L^{p}}\left\|S_{q-1} \nabla \theta\right\|_{L^{\infty}} \lesssim\|\nabla v\|_{L^{p}} \sum_{j \leq q-2} \frac{2^{j}(1+|j|)^{-\alpha}}{2^{q}(1+|q|)^{-\alpha}}\left((1+|j|)^{\alpha}\left\|\Delta_{j} \theta\right\|_{L^{\infty}}\right) .
$$

Combined with Lemma 5.2 this yields

$$
\|\mathrm{II}\|_{B_{p, r}^{0}} \lesssim\|\nabla v\|_{L^{p}}\|\theta\|_{B_{\infty, r}^{0, \alpha}} .
$$

To deal with III, we use the fact that the divergence of $\Delta_{q} v$ vanishes to write 


$$
\mathrm{III}=\sum_{q \geq 2} \mathscr{R}_{\alpha} \operatorname{div}\left(\Delta_{q} v \widetilde{\Delta}_{q} \theta\right)-\sum_{q \geq 2} \operatorname{div}\left(\Delta_{q} v \mathscr{R}_{\alpha} \widetilde{\Delta}_{q} \theta\right)+\sum_{q \leq 1}\left[\mathscr{R}_{\alpha}, \Delta_{q} v \cdot \nabla\right] \widetilde{\Delta}_{q} \theta=J_{1}+J_{2}+J_{3}
$$

Using Remark 4.2 we get

$$
\left\|\Delta_{j} \mathscr{R}_{\alpha} \operatorname{div}\left(\Delta_{q} v \widetilde{\Delta}_{q} \theta\right)\right\|_{L^{p}} \lesssim 2^{j}(1+|j|)^{\alpha}\left\|\Delta_{q} v\right\|_{L^{p}}\left\|\widetilde{\Delta}_{q} \theta\right\|_{L^{\infty}}
$$

and since $q \geq 2$,

$$
\left\|\Delta_{j} \operatorname{div}\left(\Delta_{q} v \mathscr{R}_{\alpha} \widetilde{\Delta}_{q} \theta\right)\right\|_{L^{p}} \lesssim 2^{j}\left\|\Delta_{q} v\right\|_{L^{p}}\left\|\mathscr{R}_{\alpha} \widetilde{\Delta}_{q} \theta\right\|_{L^{\infty}} \lesssim 2^{j}(1+|q|)^{\alpha}\left\|\Delta_{q} v\right\|_{L^{p}}\left\|\widetilde{\Delta}_{q} \theta\right\|_{L^{\infty}} .
$$

Therefore we get

$$
\left\|\Delta_{j}\left(J_{1}+J_{2}\right)\right\|_{L^{p}} \lesssim \sum_{\substack{q \in \mathbb{N} \\ q \geq j-4}} 2^{j}(1+|q|)^{\alpha}\left\|\Delta_{q} v\right\|_{L^{p}}\left\|\widetilde{\Delta}_{q} \theta\right\|_{L^{\infty}} \lesssim\|\nabla v\|_{L^{p}} \sum_{\substack{q \in \mathbb{N} \\ q \geq j-4}} 2^{j-q}(1+|q|)^{\alpha}\left\|\Delta_{q} \theta\right\|_{L^{\infty}}
$$

where we have again used Bernstein inequality to get the last inequality. It suffices now to use Lemma 5.2:

$$
\left\|J_{1}+J_{2}\right\|_{B_{p, r}^{0}} \lesssim\|\nabla v\|_{L^{p}}\|\theta\|_{B_{\infty, r}^{0, \alpha}}
$$

For the term $J_{3}$ we can write

$$
\sum_{-1 \leq q \leq 1}\left[\mathscr{R}_{\alpha}, \Delta_{q} v \cdot \nabla\right] \widetilde{\Delta}_{q} \theta(x)=\sum_{q \leq 1}\left[\operatorname{div} \tilde{\chi}(\mathrm{D}) \mathscr{R}_{\alpha}, \Delta_{q} v\right] \widetilde{\Delta}_{q} \theta(x),
$$

where $\tilde{\chi}$ belongs to $\mathscr{D}\left(\mathbb{R}^{d}\right)$. From the proof of Proposition 4.1 we know that $\operatorname{div} \tilde{\chi}(\mathrm{D}) \mathscr{R}_{\alpha}$ is a convolution operator with a kernel $\tilde{h}$ satisfying

$$
|\tilde{h}(x)| \lesssim(1+|x|)^{-d-1}
$$

Thus

$$
J_{3}=\sum_{q \leq 1} \tilde{h} \star\left(\Delta_{q} v \cdot \tilde{\Delta}_{q} \theta\right)-\Delta_{q} v \cdot\left(\tilde{h} \star \tilde{\Delta}_{q} \theta\right) .
$$

Note that $\Delta_{j} J_{3}=0$ for $j \geq 6$; thus we just need to estimate the low frequencies of $J_{3}$. Since $x \tilde{h}$ belongs to $L^{p^{\prime}}$ for $p^{\prime}>1$, we can use Lemma 5.1 with $m=p \geq 2$ to obtain

$$
\left\|\Delta_{j} J_{3}\right\|_{L^{p}} \lesssim \sum_{q \leq 1}\|x \tilde{h}\|_{L^{p^{\prime}}}\left\|\Delta_{q} \nabla v\right\|_{L^{p}}\left\|\widetilde{\Delta}_{q} \theta\right\|_{L^{p}} \lesssim\|\nabla v\|_{L^{p}} \sum_{-1 \leq q \leq 1}\left\|\Delta_{q} \theta\right\|_{L^{p}}
$$

This yields

$$
\left\|J_{3}\right\|_{B_{p, r}^{0}} \lesssim\|\nabla v\|_{L^{p}}\|\theta\|_{L^{p}}
$$

completing the proof of the first part of Proposition 5.3.

(2) The second part can be done similarly, so we give a shorter proof. To estimate the terms I and II we use two estimates: $\left\|\Delta_{q} \nabla u\right\|_{L^{\infty}} \approx\left\|\Delta_{q} \omega\right\|_{L^{\infty}}$ for all $q \in \mathbb{N}$, and

$$
\left\|\nabla S_{q-1} v\right\|_{L^{\infty}} \lesssim\left\|\nabla \Delta_{-1} v\right\|_{L^{\infty}}+\sum_{j=0}^{q-2}\left\|\Delta_{j} \nabla v\right\|_{L^{\infty}} \lesssim\|\omega\|_{L^{\rho}}+q\|\omega\|_{L^{\infty}}
$$

Thus (15) becomes

$$
\left\|\mathrm{I}_{q}\right\|_{L^{\infty}} \leq\|\omega\|_{L^{\infty}}(1+|q|)^{1+\alpha}\left\|\Delta_{q} \theta\right\|_{L^{\infty}}
$$


and by Corollary 4.3

$$
\|\mathrm{I}\|_{B_{\infty, r}^{0}} \leq\|\omega\|_{L^{\infty}}\|\theta\|_{B_{\infty, r}^{0,1+\alpha}} \leq\|\omega\|_{L^{\infty}}\|\theta\|_{B_{\infty, \infty}^{\epsilon}} .
$$

The second term II is estimated as

$$
\|\mathrm{II}\|_{B_{\infty, r}^{0}} \leq\|\omega\|_{L^{\infty}}\|\theta\|_{B_{\infty, r}^{0, \alpha}} \leq\|\omega\|_{L^{\infty}}\|\theta\|_{B_{\infty, \infty}^{\epsilon}} .
$$

For the remaining term the analysis is the same as before, except for $J_{3}$, where we apply Lemma 5.1 with $p=\infty$ and $m=\rho$, leading to

$$
\left\|\Delta_{j} J_{3}\right\|_{L^{p}} \lesssim \sum_{q \leq 1}\|x \tilde{h}\|_{L^{\rho^{\prime}}}\left\|\Delta_{q} \nabla v\right\|_{L^{\infty}}\left\|\widetilde{\Delta}_{q} \theta\right\|_{L^{\rho}} \lesssim\|\nabla v\|_{L^{\rho}} \sum_{-1 \leq q \leq 1}\left\|\Delta_{q} \theta\right\|_{L^{\rho}} \lesssim\|\omega\|_{L^{\rho}}\|\theta\|_{L^{\rho}} .
$$

This ends the proof of the theorem.

\section{Smoothing effects}

In this section we describe smoothing effects for the model (6), with zero source term $f$ :

$$
\left\{\begin{array}{l}
\partial_{t} \theta+v \cdot \nabla \theta+\mathscr{L} \theta=0, \\
\theta_{\mid t=0}=\theta_{0}
\end{array}\right.
$$

with

and $\operatorname{div} v=0$.

$$
\mathscr{L}:=\frac{|\mathrm{D}|^{\beta}}{\log ^{\alpha}(\lambda+|\mathrm{D}|)}
$$

Theorem 6.1. Let $\left.\left.\alpha \geq 0, \lambda \geq e^{3+2 \alpha}, d \in\{2,3\}, \beta \in\right] 0,1\right]$ and let $v$ be a smooth divergence-free vector field of $\mathbb{R}^{d}$ with vorticity $\omega$. Then, for every $\left.p \in\right] 1, \infty[$, there exists a constant $C$ such that

$$
\sup _{q \in \mathbb{N}} 2^{q \beta}(1+q)^{-\alpha}\left\|\Delta_{q} \theta\right\|_{L_{t}^{1} L^{p}} \leq C\left\|\theta_{0}\right\|_{L^{p}}+C\left\|\theta_{0}\right\|_{L^{\infty}}\|\omega\|_{L_{t}^{1} L^{p}},
$$

for every smooth solution $\theta$ of (TD).

Remark 6.2. We give the proof in the case $\beta=1$ for simplicity, but the result remains true for $\beta \in] 0,1[$. Proof of Theorem 6.1 in the case $\beta=1$. We start by localizing the equation in frequencies. for $q \geq-1$ we set $\theta_{q}:=\Delta_{q} \theta$. Then

$$
\partial_{t} \theta_{q}+v \cdot \nabla \theta_{q}+\frac{|\mathrm{D}|}{\log ^{\alpha}(\lambda+|\mathrm{D}|)} \theta_{q}=-\left[\Delta_{q}, v \cdot \nabla\right] \theta .
$$

Recall that $\theta_{q}$ is real function since the functions involved in the dyadic partition of the unity are radial. Then multiplying the above equation by $\left|\theta_{q}\right|^{p-2} \theta_{q}$, integrating by parts and using Hölder inequalities we get

$$
\frac{1}{p} \frac{d}{d t}\left\|\theta_{q}\right\|_{L^{p}}^{p}+\int_{\mathbb{R}^{2}}\left(\frac{|\mathrm{D}|}{\log ^{\alpha}(\lambda+|\mathrm{D}|)} \theta_{q}\right)\left|\theta_{q}\right|^{p-2} \theta_{q} d x \leq\left\|\theta_{q}\right\|_{L^{p}}^{p-1}\left\|\left[\Delta_{q}, v \cdot \nabla\right] \theta\right\|_{L^{p}} .
$$

Using Theorem 1.3 we get for $q \geq 0$

$$
c 2^{q}(1+q)^{-\alpha}\left\|\theta_{q}\right\|_{L^{p}}^{p} \leq \int_{\mathbb{R}^{2}}\left(\frac{|\mathrm{D}|}{\log ^{\alpha}(\lambda+|\mathrm{D}|)} \theta_{q}\right)\left|\theta_{q}\right|^{p-2} \theta_{q} d x,
$$


where $c$ depends on $p$. Inserting this estimate in the previous one we obtain

$$
\frac{1}{p} \frac{d}{d t}\left\|\theta_{q}\right\|_{L^{p}}^{p}+c 2^{q}(1+q)^{-\alpha}\left\|\theta_{q}\right\|_{L^{p}}^{p} \lesssim\left\|\theta_{q}\right\|_{L^{p}}^{p-1}\left\|\left[\Delta_{q}, v \cdot \nabla\right] \theta\right\|_{L^{p}}
$$

Thus we find

$$
\frac{d}{d t}\left\|\theta_{q}\right\|_{L^{p}}+c 2^{q}(1+q)^{-\alpha}\left\|\theta_{q}\right\|_{L^{p}} \lesssim\left\|\left[\Delta_{q}, v \cdot \nabla\right] \theta\right\|_{L^{p}} .
$$

To estimate the right side we will use the following result; see [Hmidi et al. 2011, Proposition 3.3].

$$
\left\|\left[\Delta_{q}, v \cdot \nabla\right] \theta\right\|_{L^{p}} \lesssim\|\nabla v\|_{L^{p}}\|\theta\|_{B_{\infty, \infty}^{0}} .
$$

Combined with (16) this lemma yields

$$
\begin{aligned}
\frac{d}{d t}\left(e^{c t 2^{q}(1+q)^{-\alpha}}\left\|\theta_{q}(t)\right\|_{L^{p}}\right) & \lesssim e^{c t 2^{q}(1+q)^{-\alpha}}\|\nabla v(t)\|_{L^{p}}\|\theta(t)\|_{B_{\infty, \infty}^{0}} \\
& \lesssim e^{c t 2^{q}(1+q)^{-\alpha}}\|\omega(t)\|_{L^{p}}\left\|\theta_{0}\right\|_{L^{\infty}} .
\end{aligned}
$$

To get the last line, we have used the conservation of the $L^{\infty}$ norm of $\theta$ and the classical fact that

$$
\left.\|\nabla v\|_{L^{p}} \lesssim\|\omega\|_{L^{p}} \text { for } p \in\right] 1,+\infty[.
$$

Integrating the differential inequality we get for $q \in \mathbb{N}$

$$
\left\|\theta_{q}(t)\right\|_{L^{p}} \lesssim\left\|\theta_{q}^{0}\right\|_{L^{p}} e^{-c t 2^{q}(1+q)^{-\alpha}}+\left\|\theta_{0}\right\|_{L^{\infty}} \int_{0}^{t} e^{-c(t-\tau) 2^{q}(1+q)^{-\alpha}}\|\omega(\tau)\|_{L^{p}} d \tau .
$$

Integrating in time yields

$$
2^{q}(1+q)^{-\alpha}\left\|\theta_{q}\right\|_{L_{t}^{1} L^{p}} \lesssim\left\|\theta_{q}^{0}\right\|_{L^{p}}+\left\|\theta_{0}\right\|_{L^{\infty}} \int_{0}^{t}\|\omega(\tau)\|_{L^{p}} d \tau \lesssim\left\|\theta_{0}\right\|_{L^{p}}+\left\|\theta_{0}\right\|_{L^{\infty}} \int_{0}^{t}\|\omega(\tau)\|_{L^{p}} d \tau,
$$

which is the desired result.

\section{Proof of Theorem 1.5}

Throughout this section we use the notation $\Phi_{k}$ to denote any function of the form

$$
\Phi_{k}(t)=C_{0} \underbrace{\exp (\ldots \exp }_{k \text { times }}\left(C_{0} t\right) \ldots),
$$

where $C_{0}$ depends on the relevant norms of the initial data and its value may vary from line to line up to some absolute constants. We will make frequent and tacit use of the trivial inequalities

$$
\int_{0}^{t} \Phi_{k}(\tau) d \tau \leq \Phi_{k}(t) \quad \text { and } \quad \exp \left(\int_{0}^{t} \Phi_{k}(\tau) d \tau\right) \leq \Phi_{k+1}(t) .
$$

The proof of Theorem 1.5 is done in several steps. We first give some a priori estimates for the equations (5). Next we prove uniqueness. Finally, we discuss the construction of the solutions. 
A priori estimates. Theorem 1.5 deals with critical regularities and one needs to bound the Lipschitz norm of the velocity in order to get the global persistence of the initial regularities. For this purpose we will proceed in several steps: one of the main steps is to give an $L^{\infty}$-bound of the vorticity, but due to technical difficulties related to Riesz transforms, this is not done directly. First we prove an $L^{p}$-estimate for the vorticity with $2<p<\infty$.

Proposition 7.1. Let $\alpha \in\left[0, \frac{1}{2}\right], \lambda \geq e^{3+2 \alpha}$ and $\left.p \in\right] 2, \infty\left[\right.$. Let $(v, \theta)$ be a solution of (5) with $\omega^{0} \in$ $L^{p}, \theta_{0} \in L^{p} \cap L^{\infty}$ and $\mathscr{R}_{\alpha} \theta_{0} \in L^{p}$. Then, for every $\epsilon>0$,

$$
\|\omega(t)\|_{L^{p}}+\|\theta\|_{L_{t}^{1} B_{p, 1}^{1-\epsilon}} \leq \Phi_{2}(t) .
$$

Proof. Applying the transform $\mathscr{R}_{\alpha}$ to the temperature equation we get

$$
\partial_{t} \mathscr{R}_{\alpha} \theta+v \cdot \nabla \mathscr{R}_{\alpha} \theta+\frac{|\mathrm{D}|}{\log ^{\alpha}(\lambda+|\mathrm{D}|)} \mathscr{R}_{\alpha} \theta=-\left[\mathscr{R}_{\alpha}, v \cdot \nabla\right] \theta .
$$

Since $\frac{|\mathrm{D}|}{\log ^{\alpha}(\lambda+|\mathrm{D}|)} \mathscr{R}_{\alpha}=\partial_{1}$, the function $\Gamma:=\omega+\mathscr{R}_{\alpha} \theta$ satisfies

$$
\partial_{t} \Gamma+v \cdot \nabla \Gamma=-\left[\Re_{\alpha}, v \cdot \nabla\right] \theta .
$$

According to Proposition 5.3(1), applied with $r=2$,

$$
\left\|\left[\mathscr{R}_{\alpha}, v \cdot \nabla\right] \theta\right\|_{B_{p, 2}^{0}} \lesssim\|\nabla v\|_{L^{p}}\left(\|\theta\|_{B_{\infty, 2}^{0, \alpha}}+\|\theta\|_{L^{p}}\right) .
$$

Using the classical embedding $B_{p, 2}^{0} \hookrightarrow L^{p}$ which is true only for $p \in[2, \infty)$

$$
\left\|\left[\mathscr{R}_{\alpha}, v \cdot \nabla\right] \theta\right\|_{L^{p}} \leq\|\nabla v\|_{L^{p}}\left(\|\theta\|_{B_{\infty, 2}^{0, \alpha}}+\|\theta\|_{L^{p}}\right) .
$$

Since $\operatorname{div} v=0$, the $L^{p}$ estimate applied to the transport equation (18) gives

$$
\|\Gamma(t)\|_{L^{p}} \leq\left\|\Gamma^{0}\right\|_{L^{p}}+\int_{0}^{t}\left\|\left[\Re_{\alpha}, v \cdot \nabla\right] \theta(\tau)\right\|_{L^{p}} d \tau .
$$

Applying Theorem 3.1 to (17) yields

$$
\left\|\mathscr{R}_{\alpha} \theta(t)\right\|_{L^{p}} \leq\left\|\mathscr{R}_{\alpha} \theta_{0}\right\|_{L^{p}}+\int_{0}^{t}\left\|\left[\mathscr{R}_{\alpha}, v \cdot \nabla\right] \theta(\tau)\right\|_{L^{p}} d \tau .
$$

Set $f(t):=\|\omega(t)\|_{L^{p}}+\left\|\Re_{\alpha} \theta(t)\right\|_{L^{p}}$. From the previous estimates we get

$$
\begin{aligned}
f(t) & \lesssim\left\|\Gamma_{0}\right\|_{L^{p}}+\left\|\mathscr{R}_{\alpha} \theta_{0}\right\|_{L^{p}}+\int_{0}^{t}\|\nabla v(\tau)\|_{L^{p}}\left(\|\theta(\tau)\|_{B_{\infty, 2}^{0, \alpha}}+\|\theta\|_{L^{p}}\right) d \tau \\
& \lesssim f(0)+\int_{0}^{t} f(\tau)\left(\|\theta(\tau)\|_{B_{\infty, 2}^{0, \alpha}}+\left\|\theta_{0}\right\|_{L^{p}}\right) d \tau .
\end{aligned}
$$

Here we have used the Calderón-Zygmund estimate, to the effect that $\|\nabla v\|_{L^{p}} \leq C\|\omega\|_{L^{p}}$ for $p \in(1, \infty)$, and also the estimate $\|\theta(t)\|_{L^{p}} \leq\left\|\theta_{0}\right\|_{L^{p}}$ from Theorem 3.1.

According to Gronwall's lemma we get

$$
f(t) \lesssim f(0) e^{C\left\|\theta_{0}\right\|_{L^{p}} t} e^{C\|\theta\|_{L_{t}^{1} B_{\infty, 2}^{0, \alpha}}}
$$


For $N \in \mathbb{N}$, the Bernstein inequalities and Theorem 3.1 give

$$
\begin{aligned}
\|\theta\|_{L_{t}^{1} B_{\infty, 2}^{0, \alpha}} & \leq t\left\|\left(\sum_{q<N}(1+|q|)^{2 \alpha}\left\|\Delta_{q} \theta\right\|_{L^{\infty}}^{2}\right)^{1 / 2}\right\|\left\|_{L_{t}^{\infty}}+\right\|\left(\mathrm{Id}-S_{N}\right) \theta \|_{L_{t}^{1} B_{\infty, 1}^{0, \alpha}} \\
& \lesssim t\|\theta\|_{L_{t, x}^{\infty}} N^{1 / 2+\alpha}+\sum_{q \geq N}(1+|q|)^{\alpha}\left\|\Delta_{q} \theta\right\|_{L_{t}^{1} L^{\infty}} \\
& \lesssim t\left\|\theta_{0}\right\|_{L^{\infty}} N^{1 / 2+\alpha}+\sum_{q \geq N}(1+|q|)^{\alpha}\left\|\Delta_{q} \theta\right\|_{L_{t}^{1} L^{\infty}} \\
& \lesssim N^{1 / 2+\alpha}\left\|\theta_{0}\right\|_{L^{\infty}} t+\sum_{q \geq N} 2^{q(2 / p)}(1+|q|)^{\alpha}\left\|\Delta_{q} \theta\right\|_{L_{t}^{1} L^{p}} .
\end{aligned}
$$

Using Theorem 6.1, we obtain for $p>2$ and $0<\varepsilon<1-2 / p$

$$
\begin{aligned}
\sum_{q \geq N}(1+|q|)^{\alpha} 2^{q(2 / p)}\left\|\Delta_{q} \theta\right\|_{L_{t}^{1} L^{p}} & \lesssim \sum_{q \geq N}(1+|q|)^{2 \alpha} 2^{q((2 / p)-1)}\left(\left\|\theta_{0}\right\|_{L^{p}}+\left\|\theta_{0}\right\|_{L^{\infty}}\|\omega\|_{L_{t}^{1} L^{p}}\right) \\
& \lesssim \sum_{q \geq N} 2^{q((2 / p)+\varepsilon-1)}\left(\left\|\theta_{0}\right\|_{L^{p}}+\left\|\theta_{0}\right\|_{L^{\infty}}\|\omega\|_{L_{t}^{1} L^{p}}\right) \\
& \lesssim\left\|\theta_{0}\right\|_{L^{p}}+2^{N(-1+\varepsilon+2 / p)}\left\|\theta_{0}\right\|_{L^{\infty}}\|\omega\|_{L_{t}^{1} L^{p}} .
\end{aligned}
$$

Consequently,

$$
\|\theta\|_{L_{t}^{1} B_{\infty, 2}^{0, \alpha}} \lesssim N^{(1 / 2)+\alpha}\left\|\theta_{0}\right\|_{L^{\infty}} t+\left\|\theta_{0}\right\|_{L^{p}}+2^{N(-1+\varepsilon+2 / p)}\left\|\theta_{0}\right\|_{L^{\infty}}\|\omega\|_{L_{t}^{1} L^{p}} .
$$

We choose

$$
N=\left[\frac{\log \left(e+\|\omega\|_{L_{t}^{1} L^{p}}\right)}{(1-\varepsilon-2 / p) \log 2}\right]
$$

This yields

$$
\|\theta\|_{L_{t}^{1} B_{\infty, 2}^{0, \alpha}} \lesssim\left\|\theta_{0}\right\|_{L^{\infty} \cap L^{p}}+\left\|\theta_{0}\right\|_{L^{\infty}} t \log ^{(1 / 2)+\alpha}\left(e+\int_{0}^{t}\|\omega(\tau)\|_{L^{p}} d \tau\right) .
$$

Combining this estimate with (19) we get

$$
\begin{aligned}
\|\theta\|_{L_{t}^{1} B_{\infty, 2}^{0, \alpha}} & \lesssim\left\|\theta_{0}\right\|_{L^{\infty} \cap L^{p}}+\left\|\theta_{0}\right\|_{L^{\infty}} t \log ^{(1 / 2)+\alpha}\left(e+C f(0) e^{C\left\|\theta_{0}\right\|_{L^{p} t}} e^{C\|\theta\|_{L_{t}^{1} B_{\infty, 2}^{0, \alpha}}}\right) \\
& \leq C_{0} \log ^{(1 / 2)+\alpha}(e+f(0))\left(1+t^{(3 / 2)+\alpha}\right)+C\left\|\theta_{0}\right\|_{L^{\infty} t\|\theta\|_{L_{t}^{1} B_{\infty, 2}^{0, \alpha}}^{(1 / 2)+\alpha},}
\end{aligned}
$$

where $C_{0}$ is a constant depending on $\left\|\theta_{0}\right\|_{L^{p} \cap L^{\infty}}$.

Case 1: $\alpha<\frac{1}{2}$.

Lemma 7.2. There exists a number $C$, depending only on $\alpha \in\left[0,1\left[\right.\right.$, such that if $a, b>0$ and if $x \in \mathbb{R}_{+}$ is a solution of the inequality

$$
x \leq a+b x^{\alpha},
$$

then

$$
x \leq C\left(a+b^{1 /(1-\alpha)}\right) .
$$


Proof. Set $y=a^{-1} x$. Then (21) becomes

$$
y \leq 1+b a^{\alpha-1} y^{\alpha} .
$$

We will look for a number $\mu>0$ such that $y \leq e^{\mu}$. It suffices to find $\mu$ such that

$$
1+b a^{\alpha-1} e^{\mu \alpha} \leq e^{\mu} .
$$

In particular (since $e^{\mu \alpha}>1$ ) we can take for $\mu$ the solution of

$$
\left(1+b a^{\alpha-1}\right) e^{\mu \alpha}=e^{\mu} .
$$

This gives $e^{\mu}=\left(1+b a^{\alpha-1}\right)^{1 /(1-\alpha)}$. Now recall that there is a constant $C=C_{\alpha}$ such that, for every $t, s \geq 0$,

$$
(t+s)^{1 /(1-\alpha)} \leq C\left(t^{1 /(1-\alpha)}+s^{1 /(1-\alpha)}\right) .
$$

With this constant we have $y \leq C\left(1+b^{1 /(1-\alpha)} a^{-1}\right)$, or equivalently $x \leq C\left(a+b^{1 /(1-\alpha)}\right)$, as required.

Applying this lemma to (20) we get, for every $t \in \mathbb{R}_{+}$,

$$
\|\theta\|_{L_{t}^{1} B_{\infty, 2}^{0, \alpha}} \leq C_{0}\left(t^{3 / 2}+t^{2 /(1-2 \alpha)}\right) \leq C_{0}\left(1+t^{2 /(1-2 \alpha)}\right) \leq \Phi_{1}(t) .
$$

It follows from (19) that

$$
f(t) \leq C_{0} e^{C_{0} t^{2 /(1-2 \alpha)}} \leq \Phi_{2}(t)
$$

Applying Theorem 6.1 and (23) we get, for every $\epsilon>0$ and $q \in \mathbb{N}$,

$$
2^{q}(1+|q|)^{-\alpha}\left\|\Delta_{q} \theta\right\|_{L_{t}^{1} L^{p}} \leq C_{0} e^{C_{0} t^{2 /(1-2 \alpha)}} \leq \Phi_{2}(t) .
$$

Case 2: $\alpha=\frac{1}{2}$. The estimate (20) becomes

$$
\|\theta\|_{L_{t}^{1} B_{\infty, 2}^{0,1 / 2}} \leq C_{0} \log (e+f(0))\left(1+t^{2}\right)+C\left\|\theta_{0}\right\|_{L^{\infty}} t\|\theta\|_{L_{t}^{1} B_{\infty, 2}^{0,1 / 2}},
$$

with $C_{0}$ depending on $\left\|\theta_{0}\right\|_{L^{p} \cap L^{\infty}}$. Hence if we choose $t$ small enough that

$$
C\left\|\theta_{0}\right\|_{L^{\infty}}=\frac{1}{2}
$$

then

$$
\|\theta\|_{L_{t}^{1} B_{\infty, 2}^{0, \alpha}} \leq C_{0} \log (e+f(0))
$$

From (19) we get that

$$
f(t) \leq C_{0}(e+f(0))^{C_{0}} .
$$

Now let $t$ be a given positive time and choose a partition $\left(t_{i}\right)_{i=1}^{N}$ of $[0, t]$ such that

$$
C\left\|\theta_{0}\right\|_{L^{\infty}}\left(t_{i+1}-t_{i}\right) \approx \frac{1}{2} .
$$

Set $a_{i}:=\int_{t_{i}}^{t_{i+1}}\|\theta(\tau)\|_{B_{\infty, 2}^{0,1 / 2}} d \tau$ and $b_{i}=f\left(t_{i}\right)$. Computations similar to (20) yield

$$
a_{i} \leq C_{0} \log \left(e+b_{i}\right)\left(1+\left(t_{i+1}-t_{i}\right)^{2}\right)+C\left\|\theta_{0}\right\|_{L^{\infty}}\left(t_{i+1}-t_{i}\right) a_{i} .
$$

Hence we get

$$
a_{i} \leq C_{0} \log \left(e+b_{i}\right) \text {. }
$$


The analogous estimate to (19) is

$$
b_{i+1} \lesssim b_{i} e^{C\left(t_{i+1}-t_{i}\right)\left\|\theta_{0}\right\|_{L^{p}}} e^{C a_{i}} \leq C_{0} b_{i} e^{C a_{i}} .
$$

Combining (26) and (27) yields

$$
b_{i+1} \leq C_{0}\left(e+b_{i}\right)^{C_{0}} .
$$

By induction we can prove that for every $i \in\{1, \ldots, N\}$ we have $b_{i} \leq C_{0} e^{\exp C_{0} i}$, and consequently, from (26), $a_{i} \leq C_{0} e^{C_{0} i}$. It follows that

$$
\|\theta\|_{L_{t}^{1} B_{\infty, 2}^{0,1 / 2}}=\sum_{i=1}^{N} a_{i} \leq C_{0} e^{C_{0} N} \leq C_{0} e^{C_{0} t} .
$$

We have used in the last inequality the fact that $N \approx C_{0} t$, a consequence of (25). We have also obtained

$$
f(t) \leq C_{0} e^{\exp C_{0} t}
$$

It is not hard to see that (24) implies

$$
\|\theta\|_{L_{t}^{1} B_{p, 1}^{s}} \leq\|\theta\|_{\widetilde{L}_{t}^{1} B_{p, \infty}^{1,-\alpha}} \leq \Phi_{2}(t) \quad \text { for every } s<1 .
$$

This ends the proof of Proposition 7.1.

Remark 7.3. Combining (28) with the Bernstein inequalities and the fact that $p>2$ yields

$$
\|\theta\|_{L_{t}^{1} B_{\infty, 1}^{\epsilon}} \leq \Phi_{2}(t) \text { for every } \epsilon<1-\frac{2}{p} .
$$

We are now ready to prove an $L^{\infty}$-bound on the vorticity.

Proposition 7.4. Let $\left.\alpha \in\left[0, \frac{1}{2}\right], \lambda \geq e^{3+2 \alpha}, p \in\right] 2, \infty[$, and let $(v, \theta)$ be a smooth solution of the system (5) such that $\omega^{0}, \theta_{0}, \mathscr{R}_{\alpha} \theta_{0} \in L^{p} \cap L^{\infty}$. Then we have

$$
\|\omega(t)\|_{L^{\infty}}+\left\|\mathscr{R}_{\alpha} \theta(t)\right\|_{L^{\infty}} \leq \Phi_{3}(t)
$$

and

$$
\|v(t)\|_{L^{\infty}} \leq \Phi_{4}(t)
$$

Proof of (30). By using the maximum principle for the transport equation (18), we get

$$
\|\Gamma(t)\|_{L^{\infty}} \leq\left\|\Gamma^{0}\right\|_{L^{\infty}}+\int_{0}^{t}\left\|\left[\mathscr{R}_{\alpha}, v \cdot \nabla\right] \theta(\tau)\right\|_{L^{\infty}} d \tau .
$$

Since the function $\mathscr{R}_{\alpha} \theta$ satisfies the equation

$$
\left(\partial_{t}+v \cdot \nabla+|\mathrm{D}| \log ^{-\alpha}(\lambda+|\mathrm{D}|)\right) \mathscr{R}_{\alpha} \theta=-\left[\mathscr{R}_{\alpha}, v \cdot \nabla\right] \theta,
$$

we get, using Theorem 3.1,

$$
\left\|\mathscr{R}_{\alpha} \theta(t)\right\|_{L^{\infty}} \leq\left\|\mathscr{R}_{\alpha} \theta(t)\right\|_{L^{\infty}}+\int_{0}^{t}\left\|\left[\mathscr{R}_{\alpha}, v \cdot \nabla\right] \theta(\tau)\right\|_{L^{\infty}} d \tau .
$$


Thus we obtain

$$
\begin{aligned}
\|\Gamma(t)\|_{L^{\infty}}+\left\|\mathscr{R}_{\alpha} \theta(t)\right\|_{L^{\infty}} & \leq\left\|\Gamma^{0}\right\|_{L^{\infty}}+\left\|\mathscr{R}_{\alpha} \theta_{0}\right\|_{L^{\infty}}+2 \int_{0}^{t}\left\|\left[\mathscr{R}_{\alpha}, v \cdot \nabla\right] \theta(\tau)\right\|_{L^{\infty}} d \tau \\
& \leq C_{0}+\int_{0}^{t}\left\|\left[\mathscr{R}_{\alpha}, v \cdot \nabla\right] \theta(\tau)\right\|_{B_{\infty, 1}^{0}} d \tau
\end{aligned}
$$

It follows from Theorem 3.1, Proposition 5.3(2) and Proposition 7.1 that $\|\omega(t)\|_{L^{\infty}}+\left\|\mathscr{R}_{\alpha} \theta(t)\right\|_{L^{\infty}}$

$$
\begin{aligned}
& \lesssim C_{0}+\int_{0}^{t}\|\omega(\tau)\|_{L^{\infty} \cap L^{p}}\left(\|\theta(\tau)\|_{B_{\infty, 1}^{\epsilon}}+\|\theta(\tau)\|_{L^{p}}\right) d \tau \\
& \lesssim C_{0}+\|\omega\|_{L_{t}^{\infty} L^{p}}\left(\|\theta\|_{L_{t}^{1} B_{\infty, 1}^{\epsilon}}+t\left\|\theta_{0}\right\|_{L^{p}}\right)+\int_{0}^{t}\|\omega(\tau)\|_{L^{\infty}}\left(\|\theta(\tau)\|_{B_{\infty, 1}^{\epsilon}}+\left\|\theta_{0}\right\|_{L^{p}}\right) d \tau .
\end{aligned}
$$

Let $0<\epsilon<1-\frac{2}{p}$. Using (29) we get

$$
\|\omega(t)\|_{L^{\infty}}+\left\|\mathscr{R}_{\alpha} \theta(t)\right\|_{L^{\infty}} \lesssim \Phi_{2}(t)+\int_{0}^{t}\|\omega(\tau)\|_{L^{\infty}}\left(\|\theta(\tau)\|_{B_{\infty, 1}^{\epsilon}}+\left\|\theta_{0}\right\|_{L^{p}}\right) d \tau .
$$

Therefore we obtain by the Gronwall lemma and (29)

$$
\|\omega(t)\|_{L^{\infty}}+\left\|\mathscr{R}_{\alpha} \theta(t)\right\|_{L^{\infty}} \leq \Phi_{3}(t)
$$

Proof of (31). Let $N \in \mathbb{N}$ to be chosen later. Using the fact that $\left\|\dot{\Delta}_{q} v\right\|_{L^{\infty}} \approx 2^{-q}\left\|\dot{\Delta}_{q} \omega\right\|_{L^{\infty}}$, we get

$$
\begin{aligned}
\|v(t)\|_{L^{\infty}} & \leq\left\|\chi\left(2^{N}|\mathrm{D}|\right) v(t)\right\|_{L^{\infty}}+\sum_{q \geq-N} 2^{-q}\left\|\dot{\Delta}_{q} \omega(t)\right\|_{L^{\infty}} \\
& \leq\left\|\chi\left(2^{N}|\mathrm{D}|\right) v(t)\right\|_{L^{\infty}}+2^{N}\|\omega(t)\|_{L^{\infty}} .
\end{aligned}
$$

Applying the frequency localizing operator to the velocity equation we get

$$
\chi\left(2^{N}|\mathrm{D}|\right) v=\chi\left(2^{N}|\mathrm{D}|\right) v_{0}+\int_{0}^{t} \mathscr{P} \chi\left(2^{N}|\mathrm{D}|\right) \theta(\tau) d \tau+\int_{0}^{t} \mathscr{P} \chi\left(2^{N}|\mathrm{D}|\right) \operatorname{div}(v \otimes v)(\tau) d \tau,
$$

where $\mathscr{P}$ stands for Leray projector. From Lemma 2.1, a Calderón-Zygmund estimate and the uniform boundedness of $\chi\left(2^{N}|\mathrm{D}|\right)$ we get

$$
\int_{0}^{t}\left\|\chi\left(2^{N}|\mathrm{D}|\right) \mathscr{P} \theta(\tau)\right\|_{L^{\infty}} d \tau \lesssim 2^{-N(2 / p)} \int_{0}^{t}\|\theta(\tau)\|_{L^{p}} d \tau \lesssim t\left\|\theta_{0}\right\|_{L^{p}} .
$$

Using Corollary 3.9(2) we find

$$
\int_{0}^{t}\left\|\mathscr{P} \chi\left(2^{N}|\mathrm{D}|\right) \operatorname{div}(v \otimes v)(\tau)\right\|_{L^{\infty}} d \tau \lesssim 2^{N} \int_{0}^{t}\|v(\tau)\|_{L^{\infty}}^{2} d \tau
$$

The outcome is $\|v(t)\|_{L^{\infty}} \lesssim\left\|v_{0}\right\|_{L^{\infty}}+t\left\|\theta_{0}\right\|_{L^{p}}+2^{-N} \int_{0}^{t}\|v(\tau)\|_{L^{\infty}}^{2} d \tau+2^{N}\|\omega(t)\|_{L^{\infty}} \lesssim 2^{-N} \int_{0}^{t}\|v(\tau)\|_{L^{\infty}}^{2} d \tau+2^{N} \Phi_{3}(t)$. 
Choosing judiciously $N$ we find

$$
\|v(t)\|_{L^{\infty}} \leq \Phi_{3}(t)\left(1+\left(\int_{0}^{t}\|v(\tau)\|_{L^{\infty}}^{2} d \tau\right)^{1 / 2}\right) .
$$

From the Gronwall lemma we get $\|v(t)\|_{L^{\infty}} \leq \Phi_{4}(t)$, as desired.

Finally, we turn to a Lipschitz bound of the velocity.

Proposition 7.5. Let $\left.\alpha \in\left[0, \frac{1}{2}\right], \lambda \geq e^{3+2 \alpha}, p \in\right] 2, \infty[$, and let $(v, \theta)$ be a smooth solution of the system (5) with $\omega^{0}, \theta_{0}, \mathscr{R}_{\alpha} \theta_{0} \in B_{\infty, 1}^{0} \cap L^{p}$. Then

$$
\left\|\mathscr{R}_{\alpha} \theta(t)\right\|_{B_{\infty, 1}^{0}}+\|\omega(t)\|_{B_{\infty, 1}^{0}}+\|v(t)\|_{B_{\infty, 1}^{1}} \leq \Phi_{4}(t) .
$$

Proof. Applying Corollary 3.17 to the equations (17) and (18), we obtain

$$
\|\Gamma(t)\|_{B_{\infty, 1}^{0}}+\left\|\mathscr{R}_{\alpha} \theta(t)\right\|_{B_{\infty, 1}^{0}} \lesssim\left(C_{0}+\left\|\left[\mathscr{R}_{\alpha}, v \cdot \nabla\right] \theta\right\|_{L_{t}^{1} B_{\infty, 1}^{0}}\right)\left(1+\|\nabla v\|_{L_{t}^{1} L^{\infty}}\right) .
$$

Thanks to Propositions 5.3, 7.4, 7.1 and Equation (29) we get

$$
\left\|\left[\mathscr{R}_{\alpha}, v \cdot \nabla\right] \theta\right\|_{L_{t}^{1} B_{\infty, 1}^{0}} \lesssim \int_{0}^{t}\left(\|\omega(\tau)\|_{L^{\infty}}+\|\omega(\tau)\|_{L^{p}}\right)\left(\|\theta(\tau)\|_{B_{\infty, 1}^{\epsilon}}+\|\theta(\tau)\|_{L^{p}}\right) d \tau \lesssim \Phi_{3}(t) .
$$

By easy computations we get

$$
\begin{aligned}
\|\nabla v\|_{L^{\infty}} & \leq\left\|\nabla \Delta_{-1} v\right\|_{L^{\infty}}+\sum_{q \in \mathbb{N}}\left\|\Delta_{q} \nabla v\right\|_{L^{\infty}} \lesssim\|\omega\|_{L^{p}}+\sum_{q \in \mathbb{N}}\left\|\Delta_{q} \omega\right\|_{L^{\infty}} \\
& \lesssim \Phi_{2}(t)+\|\omega(t)\|_{B_{\infty, 1}^{0}} .
\end{aligned}
$$

Putting together (32) and (33) leads to

$$
\|\omega(t)\|_{B_{\infty, 1}^{0}} \leq\|\Gamma(t)\|_{B_{\infty, 1}^{0}}+\left\|\mathscr{R}_{\alpha} \theta(t)\right\|_{B_{\infty, 1}^{0}} \leq \Phi_{3}(t)\left(1+\int_{0}^{t}\|\omega(\tau)\|_{B_{\infty, 1}^{0}} d \tau\right) .
$$

Thus we obtain from the Gronwall inequality

$$
\|\omega(t)\|_{B_{\infty, 1}^{0}}+\left\|\mathscr{R}_{\alpha} \theta(t)\right\|_{B_{\infty, 1}^{0}} \leq \Phi_{4}(t)
$$

Coming back to (33) we get

$$
\|\nabla v(t)\|_{L^{\infty}} \leq \Phi_{4}(t) .
$$

Let us move to the estimate of $v$ in the space $B_{\infty, 1}^{1}$. By definition we have

$$
\|v(t)\|_{B_{\infty, 1}^{1}} \lesssim\|v(t)\|_{L^{\infty}}+\|\omega(t)\|_{B_{\infty, 1}^{0}} .
$$

Combined with (31) and (34) this yields

$$
\|v(t)\|_{B_{\infty, 1}^{1}} \leq \Phi_{4}(t) .
$$

The proof of Proposition 7.5 is now achieved, and with it the first step in the proof of Theorem 1.5, according to outline on page 274 . 
Uniqueness. We will show that the Boussinesq system (5) has a unique solution in the function space

$$
\mathscr{\mathscr { E }}_{T}=\left(L_{T}^{\infty} B_{\infty, 1}^{0} \cap L_{T}^{1} B_{\infty, 1}^{1}\right) \times\left(L_{T}^{\infty} L^{p} \cap \widetilde{L}_{T}^{1} B_{p, \infty}^{1,-\alpha}\right), \quad 2<p<\infty .
$$

Let $\left(v^{1}, \theta^{1}\right)$ and $\left(v^{2}, \theta^{2}\right)$ be two solutions of (5) belonging to the space $\mathscr{E}_{T}$, and set

$$
v=v^{2}-v^{1}, \quad \theta=\theta^{2}-\theta^{1} .
$$

Then we get

$$
\left\{\begin{array}{l}
\partial_{t} v+v^{2} \cdot \nabla v=-\nabla \pi-v \cdot \nabla v^{1}+\theta e_{2}, \\
\partial_{t} \theta+v^{2} \cdot \nabla \theta+\frac{|\mathrm{D}|}{\log ^{\alpha}(\lambda+|\mathrm{D}|)} \theta=-v \cdot \nabla \theta^{1}, \\
v_{\mid t=0}=v_{0}, \quad \theta_{\mid t=0}=\theta_{0} .
\end{array}\right.
$$

According to Proposition 3.15 we have

$$
\|v(t)\|_{B_{\infty, 1}^{0}} \leq C e^{C V_{1}(t)}\left(\left\|v_{0}\right\|_{B_{\infty, 1}^{0}}+\|\nabla \pi\|_{L_{t}^{1} B_{\infty, 1}^{0}}+\left\|v \cdot \nabla v^{1}\right\|_{L_{t}^{1} B_{\infty, 1}^{0}}+\|\theta\|_{L_{t}^{1} B_{\infty, 1}^{0}}\right),
$$

with $V_{1}(t)=\left\|\nabla v^{1}\right\|_{L_{t}^{1} L^{\infty}}$. Straightforward computations using the incompressibility of the flows gives

$$
\begin{aligned}
\nabla \pi & =-\nabla \Delta^{-1} \operatorname{div}\left(v \cdot \nabla\left(v^{1}+v^{2}\right)\right)+\nabla \Delta^{-1} \partial_{2} \theta \\
& =\mathrm{I}+\mathrm{II} .
\end{aligned}
$$

To estimate the term I we use the definition

$$
\|\mathrm{I}\|_{B_{\infty, 1}^{0}} \lesssim\left\|\left(\nabla \Delta^{-1} \operatorname{div}\right) \operatorname{div} \Delta_{-1}\left(v \otimes\left(v^{1}+v^{2}\right)\right)\right\|_{L^{\infty}}+\left\|v \cdot \nabla\left(v^{1}+v^{2}\right)\right\|_{B_{\infty, 1}^{1}} .
$$

From Proposition 3.1(2) of [Hmidi et al. 2011] and Besov embeddings we have

$$
\left\|\left(\nabla \Delta^{-1} \operatorname{div}\right) \operatorname{div} \Delta_{-1}\left(v \otimes\left(v^{1}+v^{2}\right)\right)\right\|_{L^{\infty}} \lesssim\left\|v \otimes\left(v^{1}+v^{2}\right)\right\|_{L^{\infty}} \lesssim\|v\|_{B_{\infty, 1}^{0}}\left\|v^{1}+v^{2}\right\|_{B_{\infty, 1}^{0}} .
$$

Using the incompressibility of $v$ and Bony's decomposition one can easily obtain

$$
\left\|v \cdot \nabla\left(v^{1}+v^{2}\right)\right\|_{B_{\infty, 1}^{0}} \lesssim\|v\|_{B_{\infty, 1}^{0}}\left\|v^{1}+v^{2}\right\|_{B_{\infty, 1}^{1}} .
$$

Putting together these estimates yields

$$
\|\mathrm{I}\|_{B_{\infty, 1}^{0}} \lesssim\|v\|_{B_{\infty, 1}^{0}}\left\|v^{1}+v^{2}\right\|_{B_{\infty, 1}^{1}}
$$

We now turn to the term II. By using Besov embeddings and a Calderón-Zygmund estimate we get

$$
\|\mathrm{II}\|_{B_{\infty, 1}^{0}} \lesssim\left\|\nabla \Delta^{-1} \partial_{2} \theta\right\|_{B_{p, 1}^{2 / p}} \lesssim\|\theta\|_{B_{p, 1}^{2 / p}}
$$

Combining this estimate with (35) yields

$$
\|v(t)\|_{B_{\infty, 1}^{0}} \lesssim e^{C V(t)}\left(\left\|v_{0}\right\|_{B_{\infty, 1}^{0}}+\int_{0}^{t}\|v(\tau)\|_{B_{\infty, 1}^{0}}\left(1+\left\|\left(v^{1}, v^{2}\right)(\tau)\right\|_{B_{\infty, 1}^{1}}\right) d \tau\right)+e^{C V(t)}\|\theta\|_{L_{t}^{1} B_{p, 1}^{2 / p}}
$$

where $V(t):=\left\|\left(v^{1}, v^{2}\right)\right\|_{L_{t}^{1} B_{\infty, 1}^{1}}$. 
Now we have to estimate $\|\theta\|_{L_{t}^{1} B_{p, 1}^{2 / p}}$. By applying $\Delta_{q}$ to the equation of $\theta$ and arguing similarly to the proof of Theorem 6.1 we obtain for $q \in \mathbb{N}$

$$
\begin{aligned}
\left\|\theta_{q}(t)\right\|_{L^{p}} \lesssim e^{-c t 2^{q}(1+q)^{-\alpha}}\left\|\theta_{q}^{0}\right\|_{L^{p}}+\int_{0}^{t} e^{-c 2^{q}(1+q)^{-\alpha}(t-\tau)} & \left\|\Delta_{q}\left(v \cdot \nabla \theta^{1}\right)(\tau)\right\|_{L^{p}} d \tau \\
& +\int_{0}^{t} e^{-c 2^{q}(1+q)^{-\alpha}(t-\tau)}\left\|\left[v^{2} \cdot \nabla, \Delta_{q}\right] \theta(\tau)\right\|_{L^{p}} d \tau .
\end{aligned}
$$

Remark, first, that an obvious Hölder inequality yields that for every $\varepsilon \in[0,1]$ there exists an absolute constant $C$ such that

$$
\int_{0}^{t} e^{-c \tau 2^{q}(1+q)^{-\alpha}} d \tau \leq C t^{1-\varepsilon} 2^{-q \varepsilon}(1+q)^{\alpha \varepsilon} \quad \text { for all } t \geq 0
$$

Using this fact and integrating in time we obtain

$$
2^{q 2 / p}\left\|\theta_{q}\right\|_{L_{t}^{1} L^{p}} \lesssim(q+1)^{\alpha} 2^{q(-1+2 / p)}\left\|\theta_{q}^{0}\right\|_{L^{p}}+\mathrm{I}_{q}(t)+\mathrm{II}_{q}(t)
$$

where

$$
\begin{gathered}
\mathrm{I}_{q}(t)=t^{1-\varepsilon}(q+1)^{\alpha \varepsilon} 2^{q(-\varepsilon+2 / p)} \int_{0}^{t}\left\|\Delta_{q}\left(v \cdot \nabla \theta^{1}\right)(\tau)\right\|_{L^{p}} d \tau, \\
\mathrm{II}_{q}(t)=t^{1-\varepsilon}(q+1)^{\alpha \varepsilon} 2^{q(-\varepsilon+2 / p)} \int_{0}^{t}\left\|\left[v^{2} \cdot \nabla, \Delta_{q}\right] \theta(\tau)\right\|_{L^{p}} d \tau .
\end{gathered}
$$

Using Bony's decomposition we get easily

$$
\begin{aligned}
&\left\|\Delta_{q}\left(v \cdot \nabla \theta^{1}\right)(t)\right\|_{L^{p}} \lesssim\|v(t)\|_{L^{\infty}} \sum_{j \leq q+2} 2^{j}\left\|\Delta_{j} \theta^{1}(t)\right\|_{L^{p}}+2^{q}\|v(t)\|_{L^{\infty}} \sum_{j \geq q-4}\left\|\Delta_{j} \theta^{1}(t)\right\|_{L^{p}} \\
& \lesssim\|v(t)\|_{L^{\infty}} \sum_{j \leq q+2}(1+|j|)^{\alpha}\left(2^{j}(1+|j|)^{-\alpha}\left\|\Delta_{j} \theta^{1}(t)\right\|_{L^{p}}\right) \\
&+\|v(t)\|_{L^{\infty}} \sum_{j \geq q-4} 2^{q-j}(1+|j|)^{\alpha}\left(2^{j}(1+|j|)^{-\alpha}\left\|\Delta_{j} \theta^{1}(t)\right\|_{L^{p}}\right) .
\end{aligned}
$$

Integrating in time we get

$$
\begin{aligned}
\mathrm{I}_{q}(t) \lesssim & t^{1-\varepsilon}\|v\|_{L_{t}^{\infty} L^{\infty}} 2^{q((2 / p)-\varepsilon)}(q+1)^{1+\alpha(1+\varepsilon)}\left\|\theta^{1}\right\|_{\widetilde{L}_{t}^{1} B_{p, \infty}^{1,-\alpha}} \\
& \quad+t^{1-\varepsilon}\|v\|_{L_{t}^{\infty} L^{\infty}\left\|\theta^{1}\right\| \widetilde{L}_{t}^{1} B_{p, \infty}^{1,-\alpha}} 2^{q((2 / p)+1-\varepsilon)}(q+1)^{\alpha(1+\varepsilon)} \sum_{j \geq q-4} 2^{-j}(1+|j|)^{\alpha} \\
& \lesssim t^{1-\varepsilon}\|v\|_{L_{t}^{\infty} L^{\infty}} 2^{q((2 / p)-\varepsilon)}(q+1)^{1+\alpha(1+\varepsilon)}\left\|\theta^{1}\right\|_{\widetilde{L}_{t}^{1} B_{p, \infty}^{1,-\alpha}} .
\end{aligned}
$$

To estimate the term $\mathrm{II}_{q}$ we use the following classical commutator (since $\frac{2}{p}<1$ ) [Chemin 1998]:

$$
\left\|\left[v^{2} \cdot \nabla, \Delta_{q}\right] \theta\right\|_{L^{p}} \lesssim 2^{-q(2 / p)}\left\|\nabla v^{2}\right\|_{L^{\infty}}\|\theta\|_{B_{p, 1}^{2 / p}}
$$

Thus we obtain

$$
\mathrm{II}_{q}(t) \lesssim t^{1-\varepsilon}(q+1)^{\alpha \varepsilon} 2^{-q \varepsilon}\left\|\nabla v^{2}\right\|_{L_{t}^{\infty} L^{\infty}\|\theta\|_{L_{t}^{1} B_{p, 1}^{2 / p}}}
$$


We choose $\varepsilon \in] 0,1\left[\right.$ such that $\frac{2}{p}-\varepsilon<0$, which is possible since $p>2$. Combining (36), (37) and (38) we get

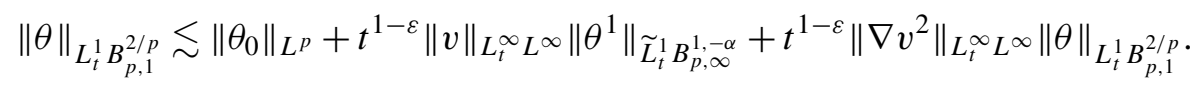

It follows that there exists a small $\delta>0$ such that for $t \in[0, \delta]$

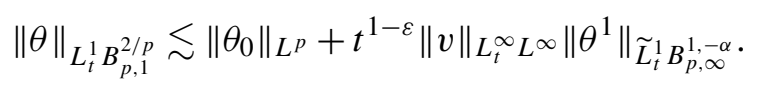

Plugging this estimate into (36) we find

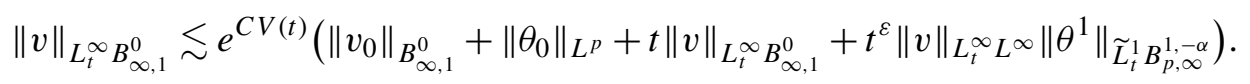

If $\delta$ is sufficiently small then we get for $t \in[0, \delta]$

$$
\|v\|_{L_{t}^{\infty} B_{\infty, 1}^{0}} \lesssim\left\|v_{0}\right\|_{B_{\infty, 1}^{0}}+\left\|\theta_{0}\right\|_{L^{p}}
$$

This gives in turn

$$
\|\theta\|_{L_{t}^{1} B_{p, 1}^{2 / p}} \lesssim\left\|v_{0}\right\|_{B_{\infty, 1}^{0}}+\left\|\theta_{0}\right\|_{L^{p}}
$$

This gives in particular the uniqueness on $[0, \delta]$. Iterating this argument yields the uniqueness in $[0, T]$.

Existence. We consider the system

$$
\left\{\begin{array}{l}
\partial_{t} v_{n}+v_{n} \cdot \nabla v_{n}+\nabla \pi_{n}=\theta_{n} e_{2}, \\
\partial_{t} \theta_{n}+v_{n} \cdot \nabla \theta_{n}+\frac{|\mathrm{D}|}{\log ^{\alpha}(\lambda+|\mathrm{D}|)} \theta_{n}=0, \\
\operatorname{div} v_{n}=0, \\
v_{n \mid t=0}=S_{n} v^{0}, \quad \theta_{n \mid t=0}=S_{n} \theta^{0} .
\end{array}\right.
$$

By using the same method as [Hmidi and Keraani 2009] we can prove that this system has a unique local smooth solution $\left(v_{n}, \theta_{n}\right)$. The global existence of these solutions is governed by the following criterion: we can push the construction beyond the time $T$ if the quantity $\left\|\nabla v_{n}\right\|_{L_{T}^{1} L^{\infty}}$ is finite. Now from the a priori estimates the Lipschitz norm cannot blow up in finite time and then the solution $\left(v_{n}, \theta_{n}\right)$ is globally defined. Once again from the a priori estimates we have for $2<p<\infty$ :

$$
\left\|v_{n}\right\|_{L_{T}^{\infty} B_{\infty, 1}^{1}}+\left\|\omega_{n}\right\|_{L_{T}^{\infty} L^{p}}+\left\|\theta_{n}\right\|_{L_{T}^{\infty} \mathscr{X}_{p}} \leq \Phi_{4}(T) .
$$

The space $\mathscr{X}_{p}$ was introduced before the statement of Theorem 1.5. It follows that up to an extraction the sequence $\left(v_{n}, \theta_{n}\right)$ is weakly convergent to $(v, \theta)$ belonging to $L_{T}^{\infty} B_{\infty, 1}^{1} \times L_{T}^{\infty} \mathscr{x}_{p}$, with $\omega \in L_{T}^{\infty} L^{p}$. For $(n, m) \in \mathbb{N}^{2}$ we set $v_{n, m}=v_{n}-v_{m}$ and $\theta_{n, m}=\theta_{n}-\theta_{m}$ then according to the estimate (39) and (40) we get for $T=\delta$,

$$
\left\|v_{n, m}\right\|_{L_{T}^{\infty} B_{\infty, 1}^{0}}+\left\|\theta_{n, m}\right\|_{L_{T}^{1} B_{p, 1}^{2 / p}} \lesssim\left\|S_{n} v_{0}-S_{m} v_{0}\right\|_{B_{\infty, 1}^{0}}+\left\|S_{n} \theta_{0}-S_{m} \theta_{0}\right\|_{L^{p}}
$$

This shows that $\left(v_{n}, \theta_{n}\right)$ is a Cauchy sequence in the Banach space $L_{T}^{\infty} B_{\infty, 1}^{0} \times L_{T}^{1} B_{p, 1}^{2 / p}$ and then it converges strongly to $(v, \theta)$. This allows to pass to the limit in the system $\left(\mathrm{B}_{n}\right)$ and then we get that $(v, \theta)$ is a solution of the Boussinesq system (5). 


\section{References}

[Askey 1973] R. Askey, "Radial characteristic functions", MRC technical summary report, no. 1262., Mathematics Research Center, University of Wisconsin-Madison, 1973.

[Beale et al. 1984] J. T. Beale, T. Kato, and A. Majda, "Remarks on the breakdown of smooth solutions for the 3-D Euler equations", Comm. Math. Phys. 94:1 (1984), 61-66. MR 85j:35154 Zbl 0573.76029

[Bochner 1959] S. Bochner, Lectures on Fourier integrals; with an author's supplement on monotonic functions, Stieltjes integrals, and harmonic analysis, Annals of Math. Studies 42, Princeton University Press, 1959. MR 21 \#5851 Zbl 0085.31802

[Bony 1981] J.-M. Bony, "Calcul symbolique et propagation des singularités pour les équations aux dérivées partielles non linéaires", Ann. Sci. École Norm. Sup. (4) 14:2 (1981), 209-246. MR 84h:35177 Zbl 0495.35024

[Chae 2006] D. Chae, "Global regularity for the 2D Boussinesq equations with partial viscosity terms", Adv. Math. 203:2 (2006), 497-513. MR 2007e:35223 Zbl 1100.35084

[Chemin 1998] J.-Y. Chemin, Perfect incompressible fluids, Oxford Lecture Series in Mathematics and its Applications 14, Oxford University Press, New York, 1998. MR 2000a:76030 Zbl 0927.76002

[Chen et al. 2007] Q. Chen, C. Miao, and Z. Zhang, “A new Bernstein's inequality and the 2D dissipative quasi-geostrophic equation”, Comm. Math. Phys. 271:3 (2007), 821-838. MR 2008b:35206 Zbl 1142.35069

[Córdoba and Córdoba 2004] A. Córdoba and D. Córdoba, "A maximum principle applied to quasi-geostrophic equations", Comm. Math. Phys. 249:3 (2004), 511-528. MR 2005f:76011 Zbl 02158321

[Danchin 2001] R. Danchin, "Local theory in critical spaces for compressible viscous and heat-conductive gases", Comm. Partial Differential Equations 26:7-8 (2001), 1183-1233. MR 2002g:76091 Zbl 1007.35071

[Danchin and Paicu 2008] R. Danchin and M. Paicu, "Existence and uniqueness results for the Boussinesq system with data in Lorentz spaces”, Phys. D 237:10-12 (2008), 1444-1460. MR 2009h:76184 Zbl 1143.76432

[Danchin and Paicu 2009] R. Danchin and M. Paicu, "Global well-posedness issues for the inviscid Boussinesq system with Yudovich's type data”, Comm. Math. Phys. 290:1 (2009), 1-14. MR 2010f:35298 Zbl 1186.35157

[Gneiting 2001] T. Gneiting, "Criteria of Pólya type for radial positive definite functions", Proc. Amer. Math. Soc. 129:8 (2001), 2309-2318. MR 2002b:42018 Zbl 1008.42012

[Hmidi and Keraani 2008] T. Hmidi and S. Keraani, "Incompressible viscous flows in borderline Besov spaces", Arch. Ration. Mech. Anal. 189:2 (2008), 283-300. MR 2009j:35252 Zbl 1147.76014

[Hmidi and Keraani 2009] T. Hmidi and S. Keraani, "On the global well-posedness of the Boussinesq system with zero viscosity”, Indiana Univ. Math. J. 58:4 (2009), 1591-1618. MR 2542974 Zbl 1178.35303

[Hmidi and Zerguine 2010] T. Hmidi and M. Zerguine, "On the global well-posedness of the Euler-Boussinesq system with fractional dissipation”, Physica D 239 (2010), 1387-1401.

[Hmidi et al. 2010] T. Hmidi, S. Keraani, and F. Rousset, "Global well-posedness for a Boussinesq-Navier-Stokes system with critical dissipation”, J. Differential Equations 249:9 (2010), 2147-2174. MR 2011j:35185 Zbl 1200.35228

[Hmidi et al. 2011] T. Hmidi, S. Keraani, and F. Rousset, "Global well-posedness for Euler-Boussinesq system with critical dissipation", Comm. Partial Differential Equations 36:3 (2011), 420-445. MR 2763332

[Hou and Li 2005] T. Y. Hou and C. Li, "Global well-posedness of the viscous Boussinesq equations", Discrete Contin. Dyn. Syst. 12:1 (2005), 1-12. MR 2005j:76026 Zbl 02154350

[Kateb 2003] D. Kateb, "On the boundedness of the mapping $f \mapsto|f|^{\mu}, \mu>1$ on Besov spaces", Math. Nachr. 248/249 (2003), 110-128. MR 2003j:46048 Zbl 1015.46022

[Liskevich and Semenov 1996] V. A. Liskevich and Y. A. Semenov, "Some problems on Markov semigroups", pp. 163-217 in Schrödinger operators, Markov semigroups, wavelet analysis, operator algebras, edited by E. Schrohe et al., Math. Top. 11, Akademie Verlag, Berlin, 1996. MR 97g:47036 Zbl 0854.47027

[Liskevich et al. 1996] V. A. Liskevich, M. A. Perelmuter, and Y. A. Semenov, "Form-bounded perturbations of generators of sub-Markovian semigroups", Acta Appl. Math. 44:3 (1996), 353-377. MR 97g:47037 Zbl 0860.47028

[Miao and Xue 2009] C. Miao and L. Xue, "On the global well-posedness of a class of Boussinesq-Navier-Stokes suystems", 2009. arXiv 0910.0311v1 
[Planchon 2000] F. Planchon, "Sur un inégalité de type Poincaré", C. R. Acad. Sci. Paris Sér. I Math. 330:1 (2000), 21-23. MR 2000m:42013 Zbl 0953.46020

[Pólya 1949] G. Pólya, "Remarks on characteristic functions", pp. 115-123 in Proceedings of the Berkeley Symposium on Mathematical Statistics and Probability, 1945, 1946, edited by J. Neyman, University of California Press, Berkeley and Los Angeles, 1949. MR 10,463c Zbl 0038.28707

[Sickel 1999] W. Sickel, "Boundedness properties of the mapping $f \rightarrow|f|^{\mu}, 0<\mu<1$ in the framework of Besov spaces", preprint 99-17, Universität Jena, 1999.

[Tao 2009] T. Tao, "Global regularity for a logarithmically supercritical hyperdissipative Naver-Stokes equation", Anal. PDE 2:3 (2009), 361-366. MR 2603802

[Trigub 1989] R. M. Trigub, "A criterion for the characteristic function and a Pólya-type test for radial functions of several variables", Teor. Veroyatnost. i Primenen. 34:4 (1989), 805-810. In Russian; translated in Theory Probab. Appl., 34:4 (1989), 738-742. MR 91f:60033

[Vishik 1998] M. Vishik, "Hydrodynamics in Besov spaces", Arch. Ration. Mech. Anal. 145:3 (1998), 197-214. MR 2000a: 35201 Zbl 0926.35123

Received 13 Nov 2009. Accepted 18 Mar 2010.

TAOUFIK HMIDI: thmidi@univ-rennes1.fr

Institut de recherche mathématique de Rennes, Université de Rennes 1, Campus de Beaulieu, 35042 Rennes Cedex, France 


\section{Analysis \& PDE}

pjm.math.berkeley.edu/apde

EDITORS

EDITOR-IN-CHIEF

Maciej Zworski

University of California

Berkeley, USA

BOARD OF EDITORS

\begin{tabular}{|c|c|c|c|}
\hline Michael Aizenman & $\begin{array}{l}\text { Princeton University, USA } \\
\text { aizenman@math.princeton.edu }\end{array}$ & Nicolas Burq & $\begin{array}{l}\text { Université Paris-Sud 11, France } \\
\text { nicolas.burq@math.u-psud.fr }\end{array}$ \\
\hline Luis A. Caffarelli & $\begin{array}{l}\text { University of Texas, USA } \\
\text { caffarel@math.utexas.edu }\end{array}$ & un-Yung Alice Chang & $\begin{array}{l}\text { Princeton University, USA } \\
\text { chang@math.princeton.edu }\end{array}$ \\
\hline Michael Christ & $\begin{array}{l}\text { University of California, Berkeley, USA } \\
\text { mchrist@math.berkeley.edu }\end{array}$ & Charles Fefferman & $\begin{array}{l}\text { Princeton University, USA } \\
\text { cf@math.princeton.edu }\end{array}$ \\
\hline Ursula Hamenstaedt & $\begin{array}{l}\text { Universität Bonn, Germany } \\
\text { ursula@math.uni-bonn.de }\end{array}$ & Nigel Higson & $\begin{array}{l}\text { Pennsylvania State Univesity, USA } \\
\text { higson@ math.psu.edu }\end{array}$ \\
\hline Vaughan Jones & $\begin{array}{l}\text { University of California, Berkeley, USA } \\
\text { vfr@math.berkeley.edu }\end{array}$ & Herbert Koch & $\begin{array}{l}\text { Universität Bonn, Germany } \\
\text { koch@math.uni-bonn.de }\end{array}$ \\
\hline Izabella Laba & $\begin{array}{l}\text { University of British Columbia, Canada } \\
\text { ilaba@math.ubc.ca }\end{array}$ & Gilles Lebeau & $\begin{array}{l}\text { Université de Nice Sophia Antipolis, France } \\
\text { lebeau@unice.fr }\end{array}$ \\
\hline László Lempert & $\begin{array}{l}\text { Purdue University, USA } \\
\text { lempert@math.purdue.edu }\end{array}$ & Richard B. Melrose & $\begin{array}{l}\text { Massachussets Institute of Technology, USA } \\
\text { rbm@math.mit.edu }\end{array}$ \\
\hline Frank Merle & $\begin{array}{l}\text { Université de Cergy-Pontoise, France } \\
\text { Frank.Merle@u-cergy.fr }\end{array}$ & William Minicozzi II & $\begin{array}{l}\text { Johns Hopkins University, USA } \\
\text { minicozz@ math.jhu.edu }\end{array}$ \\
\hline Werner Müller & $\begin{array}{l}\text { Universität Bonn, Germany } \\
\text { mueller@math.uni-bonn.de }\end{array}$ & Yuval Peres & $\begin{array}{l}\text { University of California, Berkeley, USA } \\
\text { peres@stat.berkeley.edu }\end{array}$ \\
\hline Gilles Pisier & $\begin{array}{l}\text { Texas A\&M University, and Paris } 6 \\
\text { pisier@math.tamu.edu }\end{array}$ & Tristan Rivière & $\begin{array}{l}\text { ETH, Switzerland } \\
\text { riviere@math.ethz.ch }\end{array}$ \\
\hline Igor Rodnianski & $\begin{array}{l}\text { Princeton University, USA } \\
\text { irod@math.princeton.edu }\end{array}$ & Wilhelm Schlag & $\begin{array}{l}\text { University of Chicago, USA } \\
\text { schlag@math.uchicago.edu }\end{array}$ \\
\hline Sylvia Serfaty & $\begin{array}{l}\text { New York University, USA } \\
\text { serfaty@ cims.nyu.edu }\end{array}$ & Yum-Tong Siu & $\begin{array}{l}\text { Harvard University, USA } \\
\text { siu@math.harvard.edu }\end{array}$ \\
\hline Terence Tao & $\begin{array}{l}\text { University of California, Los Angeles, USA } \\
\text { tao@math.ucla.edu }\end{array}$ & A Michael E. Taylor & $\begin{array}{l}\text { Univ. of North Carolina, Chapel Hill, USA } \\
\text { met@math.unc.edu }\end{array}$ \\
\hline Gunther Uhlmann & $\begin{array}{l}\text { University of Washington, USA } \\
\text { gunther@math.washington.edu }\end{array}$ & András Vasy & $\begin{array}{l}\text { Stanford University, USA } \\
\text { andras@math.stanford.edu }\end{array}$ \\
\hline Virgil Voicul & $\begin{array}{l}\text { University of California, Berkeley, USA } \\
\text { dvv@math.berkeley.edu }\end{array}$ & Steven Zelditch & $\begin{array}{l}\text { Northwestern University, USA } \\
\text { zelditch@math.northwestern.edu }\end{array}$ \\
\hline
\end{tabular}

PRODUCTION

contact@msp.org

Silvio Levy, Scientific Editor

Sheila Newbery, Senior Production Editor

See inside back cover or pjm.math.berkeley.edu/apde for submission instructions.

The subscription price for 2011 is US \$120/year for the electronic version, and \$180/year for print and electronic. Subscriptions, requests for back issues from the last three years and changes of subscribers address should be sent to Mathematical Sciences Publishers, Department of Mathematics, University of California, Berkeley, CA 94720-3840, USA.

Analysis \& PDE, at Mathematical Sciences Publishers, Department of Mathematics, University of California, Berkeley, CA 94720-3840 is published continuously online. Periodical rate postage paid at Berkeley, CA 94704, and additional mailing offices.

APDE peer review and production are managed by EditFLOW ${ }^{\mathrm{TM}}$ from Mathematical Sciences Publishers.

PUBLISHED BY

mathematical sciences publishers

http://msp.org/

A NON-PROFIT CORPORATION

Typeset in IATEX

Copyright $(2011$ by Mathematical Sciences Publishers 


\section{ANALYSIS \& PDE}

\section{Volume $4 \quad$ No. $2 \quad 2011$}

On the area of the symmetry orbits of cosmological spacetimes with toroidal or hyperbolic 191 symmetry

JACQUES SMULEVICI

On a maximum principle and its application to the logarithmically critical Boussinesq system 247 TAOUFIK HMIDI

Defects in semilinear wave equations and timelike minimal surfaces in Minkowski space

ROBERT JERRARD

Well- and ill-posedness issues for energy supercritical waves

Slim Ibrahim, Mohamed Majdoub and NADER Masmoudi 